\title{
Existence of mild solutions for Sobolev-type Hilfer fractional evolution equations with boundary conditions
}

\section{Haide Gou and Baolin Li'}

"Correspondence:

ghdzxh@163.com

College of Mathematics and

Statistics, Northwest Normal

University, Lanzhou, P.R. China

\begin{abstract}
This paper is concerned with the fractional differential equations of Sobolev type with boundary conditions in a Banach space. With the help of the properties of Hilfer fractional calculus, the theory of propagation families as well as the theory of the measure of noncompactness and fixed point methods, we obtain the existence results of mild solutions for Sobolev-type fractional evolution differential equations involving the Hilfer fractional derivative. Finally, an example is presented to illustrate the main result.
\end{abstract}

MSC: 26A33; 34K30; 34K45; 35B10; 47D06

Keywords: Evolution equations; Mild solutions; Hilfer fractional derivative; Noncompact measure

\section{Introduction}

In the last decades, fractional calculus and fractional differential equations have attracted much attention; we refer to [1-17] and the references therein. It is found that many phenomena can be modeled with the help of fractional derivatives or integrals, such as fractional Brownian motion [18], anomalous diffusion [19, 20], etc. Fractional differential equations have been applied to various fields successfully, for example, physics, engineering, chemistry, aerodynamics, electrodynamics of a complex medium, polymer rheology, and they have been emerging as an important area of investigation in the last few decades; see $[1,18,21-23]$. It is a development in the theory and application of fractional differential equations with the Riemann-Liouville fractional derivative or the Caputo fractional derivative, see [24-31] and the references therein.

In recent years, Hilfer fractional differential equations have received much attention. Hilfer [9, 32, 33] proposed a generalized Riemann-Liouville fractional derivative, for short, Hilfer fractional derivative, which includes the Riemann-Liouville fractional derivative and the Caputo fractional derivative. It seems that Hilfer et al. [1,34] have initially proposed linear differential equations with the new fractional operator, the Hilfer fractional derivative, and applied operational calculus to solve such simple fractional differential equations. Thereafter, Furati et al. [4] discussed the existence and uniqueness for

(c) The Author(s) 2018. This article is distributed under the terms of the Creative Commons Attribution 4.0 International License (http://creativecommons.org/licenses/by/4.0/), which permits unrestricted use, distribution, and reproduction in any medium, provided you give appropriate credit to the original author(s) and the source, provide a link to the Creative Commons license, and indicate if changes were made. 
the general problem

$$
\begin{cases}D_{a+}^{v, \mu} u(t)=f(t, u(t)), & 0 \leq v \leq 1,0<\mu<1, t>a \\ I_{a+}^{1-\gamma} u(a+)=c, & c>0, \mu \leq \gamma=\mu+v-\mu v<1\end{cases}
$$

where $D_{0+}^{v, \mu}$ is the Hilfer fractional derivative. Next, Wang and Zhang [35] extended the above initial condition to a nonlocal boundary value problem of the form

$$
\begin{cases}D_{a+}^{v, \mu} u(t)=f(t, u(t)), & 0 \leq v \leq 1,0<\mu<1, t \in(a, b], \\ I_{a+}^{1-\gamma} u(0)=\sum_{i=1}^{m} \lambda_{i} u\left(\tau_{i}\right), & \mu \leq \gamma=\mu+v-\mu v<1, \tau_{i} \in(a, b] .\end{cases}
$$

In [36], Gao and Yu studied Hilfer integral boundary value problems for the following relaxation fractional differential equations:

$$
\begin{cases}D_{0+}^{v, \mu} u(t)=c u(t)+f(t, u(t)), & c>0,0 \leq v \leq 1,0<\mu<1, t \in(0, b], \\ I_{0+}^{1-\gamma} u(0+)=\sum_{i=1}^{m} \lambda_{i} I_{0+}^{1-\gamma} u\left(\tau_{i}\right), & \mu \leq \gamma=\mu+\nu-\mu \nu<1, \tau_{i} \in(0, b)\end{cases}
$$

involving Hilfer fractional derivatives, $0 \leq v \leq 1,0<\mu<1$, by using Mittag-Leffler functions.

In particular, Gu and Trujillo [5] investigated a class of evolution equations,

$$
\left\{\begin{array}{l}
D_{0+}^{v, \mu} u(t)=A u(t)+f(t, u(t)), \quad t \in(0, b] \\
I_{0+}^{(1-v)(1-\mu)} u(0)=u_{0}
\end{array}\right.
$$

with Hilfer fractional derivatives, by the Laplace transform and density function; they first gave the mild solution definition.

On the other hand, a Sobolev-type equation appears in a variety of physical problems such as flow of fluid through fissured rocks, thermodynamics, propagation of long waves of small amplitude and so on [37-39]. The existence result of mild solutions of fractional integrodifferential equations of Sobolev type with nonlocal condition in a separable Banach space was studied by using the theory of propagation families as well as the theory of the measures of noncompactness and the condensing maps [6]. Recently, we used the fixed point theorems combined with the theory of propagation families to discuss the existence of mild solutions for nonlinear fractional non-autonomous evolution equations of Sobolev type with delay of the form

$$
\left\{\begin{array}{l}
D_{0+}^{v, \mu}(B u(t))=A u(t)+B f\left(t, u\left(\tau_{1}(t)\right), \ldots, u\left(\tau_{m}(t)\right)\right), \quad t \in J, \\
I_{0+}^{(1-v)(1-\mu)} B u(0)=B u_{0},
\end{array}\right.
$$

where $D_{0+}^{v, \mu}$ is the Hilfer fractional derivative which will be given in the next section, $0 \leq$ $v \leq 1,0<\mu<1$, the state $u(\cdot)$ takes values in a Banach space $E$. $J=[0, b](b>0), J^{\prime}=(0, b]$. This work is based on the theory of propagation families $\{W(t)\}_{t \geq 0}$ introduced by Jin Liang and Ti-Jun Xiao [19] and the measure of noncompactness, which ensure us that it is not necessary to assume the nonlinear term $f$ satisfies a Lipschitz type condition; for more details, see [34]. 
To the best of our knowledge, there are no results about Hilfer fractional evolution differential equations of Sobolev type with boundary conditions. Motivated by the above discussion, in this paper, we use fixed point theorems combined with the theory of propagation families to discuss the existence of mild solutions for existence results for Hilfer fractional evolution differential equations of Sobolev type with boundary conditions of the form

$$
\left\{\begin{array}{c}
D_{0+}^{v, \mu}(B u(t))=A u(t)+B f\left(t, u(t), \int_{0}^{t} \rho(t, s) h(t, s, u(s)) d s\right) \\
\quad 0 \leq v \leq 1,0<\mu<1, t \in J \\
I_{0+}^{1-\gamma} B u(0)=\sum_{i=1}^{m} \lambda_{i} I_{0+}^{1-\gamma} B u\left(\tau_{i}\right) \\
\quad \mu \leq \gamma=\mu+v-\mu \nu<1, \tau_{i} \in(0, b]
\end{array}\right.
$$

where $D_{0+}^{v, \mu}$ is the Hilfer fractional derivative of order $\mu$ and type $v$, which is an interpolator between Riemann-Liouville and Caputo fractional derivatives. The operator $D_{0+}^{v, \mu}$ is a generalization of the Riemann-Liouville fractional derivative operator introduced by Hifter in $[9,32,33]$, the state $u(\cdot)$ takes values in a Banach space $E . J=[0, b](b>0), J^{\prime}=(0, b] . A$ and $B$ are closed (unbounded) linear operators with domains contained in $E$, the pair $(A, B)$ generate a propagation family $\{W(t)\}_{t \geq 0} . f:[0, b] \times E \times E \rightarrow D(B) \subset E, g: C([0, b], E) \rightarrow$ $D(B) \subset E$ are given functions to be specified later, $\rho: \Delta \rightarrow R, h: \Delta \times E \rightarrow E(\Delta=\{(t, s) \in$ $[0, b] \times[0, b]: t \geq s\}), \tau_{i}, i=1,2, \ldots, m$ are pre-fixed points satisfying $0<\tau_{1} \leq \cdots \leq \tau_{m}<b$ and $\lambda_{i}$ are real numbers. Here the existence of $B^{-1}$ is not necessarily assumed.

The rest of this paper is organized as follows: In Sect. 2, we recall some basic known results and introduce some notation. In Sect. 3, we discuss the existence theorems of mild solutions for the problem (1.1). Finally, an example will be presented to illustrate the main results.

\section{Preliminaries}

In this section, we briefly recall some basic known results which will be used in the sequel. Throughout this work, we set $J=[0, b]$, where $b>0$ is a constant. Let $E$ be a Banach space with the norm $\|\cdot\|$ and the pair $(A, B)$ generate a propagation family $\{W(t)\}_{t \geq 0}$ (see Definition 2.6). We denote by $B(E)$ the Banach space of all bounded linear operators from $E$ to $E$. For a closed and linear operator $W: D(W) \subset E \rightarrow E$, where $D(W)$ is the domain of $T$, we denote by $\rho(W)$ its resolvent set. We also denote by $C(J, E)$ the Banach space of all continuous $E$-value functions on the interval $J$ with the norm $\|u\|=\sup _{t \in J}\|u(t)\|$. Let

$$
C_{1-\gamma}(J, E)=\left\{u: J \rightarrow E \mid t^{1-\gamma} u(t) \in C(J, E)\right\}
$$

with the norm $\|\cdot\|_{C_{1-\gamma}}$ defined by

$$
\|u\|_{C_{1-\gamma}}=\sup _{0 \leq t \leq b}\left|t^{1-\gamma} u(t)\right|
$$

Evidently, $C_{1-\gamma}(J, E)$ is a Banach space. Meanwhile, $C_{1-\gamma}^{\gamma}(J, E)=\left\{f \in C_{1-\gamma}(J, E),{ }^{c} D_{0^{+}}^{\alpha} f(t) \in\right.$ $\left.C_{1-\gamma}(J, E)\right\}$.

For completeness we recall the following definitions from fractional calculus. 
Definition 2.1 The Riemann-Liouville fractional integral of order $\alpha$ of a function $f$ : $[0, \infty) \rightarrow R$ is defined as

$$
I_{0+}^{\alpha} f(t)=\frac{1}{\Gamma(\alpha)} \int_{0}^{t}(t-s)^{\alpha-1} f(s) d s, \quad t>0, \alpha>0,
$$

provided the right side is point-wise defined on $(0, \infty)$.

Definition 2.2 The Riemann-Liouville derivative of order $\alpha$ with the lower limit zero for a function $f:[0, \infty) \rightarrow R$ can be written as

$$
D_{0^{+}}^{\alpha} f(t)=\frac{1}{\Gamma(n-\alpha)} \frac{d^{n}}{d t^{n}} \int_{0}^{t} \frac{f(s)}{(t-s)^{\alpha+1-n}} d s, \quad t>0, n-1<\alpha<n .
$$

Definition 2.3 The Caputo fractional derivative of order $\alpha$ for a function $f:[0, \infty) \rightarrow R$ can be written as

$$
{ }^{c} D_{0^{+}}^{\alpha} f(t)=D_{0^{+}}^{\alpha}\left[f(t)-\sum_{k=0}^{n-1} \frac{t^{k}}{k !} f^{(k)}(0)\right], \quad t>0, n-1<\alpha<n,
$$

where $n=[\alpha]+1$ and $[\alpha]$ denotes the integer part of $\alpha$.

If $u$ is an abstract function with values in $E$, then the integrals which appear in Definitions 2.2 and 2.3 are taken in Bochner's sense.

Definition 2.4 (Hilfer fractional derivative; see [9]). The generalized Riemann-Liouville fractional derivative of order $0 \leq v \leq 1$ and $0<\mu<1$ with lower limit $a$ is defined as

$$
D_{a+}^{v, \mu} f(t)=I_{a+}^{v(1-\mu)} \frac{d}{d t} I_{a+}^{(1-\nu)(1-\mu)} f(t)
$$

for functions such that the expression on the right hand side exists.

Recently (Hilfer et al. [32]), this definition for $n-1<\mu \leq n, n \in N, 0 \leq v \leq 1$, was rewritten in a more general form:

$$
D_{a+}^{v, \mu} f(t)=I_{a_{+}}^{v(n-\mu)} \frac{d^{n}}{d t^{n}} I_{a+}^{(1-v)(n-\mu)} f(t)=I_{a+}^{v(n-\mu)} D_{a+}^{\mu+v n-\mu \nu} f(t),
$$

where $D_{a_{+}}^{\mu+v n-\mu v}$ is the Riemann-Liouville fractional derivative and $I_{a_{+}}^{v(n-\mu)}$ is the RiemannLiouville integral.

Remark 2.1 (i) When $v=0,0<\mu<1$ and $a=0$, the Hilfer fractional derivative corresponds to the classical Riemann-Liouville fractional derivative:

$$
D_{0+}^{0, \mu} f(t)=\frac{d}{d t} I_{0+}^{1-\mu} f(t)=D_{0+}^{\mu} f(t)
$$

(ii) When $v=1,0<\mu<1$ and $a=0$, the Hilfer fractional derivative corresponds to the classical Caputo fractional derivative:

$$
D_{0+}^{1, \mu} f(t)=I_{0+}^{1-\mu} \frac{d}{d t} f(t)={ }^{c} D_{0+}^{\mu} f(t) .
$$


Now, we recall the basic definitions and properties of the Kuratowski measure of noncompactness that will be used later.

Definition 2.5 ([40]) Let $E$ be a Banach space and $\Omega_{E}$ be the bounded subsets of $E$. The Kuratowski measure of noncompactness is the map $\alpha: \Omega_{E} \rightarrow[0, \infty)$ defined by (here $B \in$ $\left.\Omega_{E}\right)$

$$
\alpha(B)=\inf \left\{\varepsilon>0: B=\bigcup_{i=1}^{n} B_{i} \text { and } \operatorname{diam}\left(B_{i}\right) \leq \varepsilon \text { for } i=1, \ldots, n\right\},
$$

here $\operatorname{diam} B_{i}=\sup \left\{|x-y|: x, y \in B_{i}\right\}$.

Lemma 2.1 ([41]) Let $S$ and $T$ be bounded sets of $E$ and $a$ be a real number. Then the noncompactness measure has the following properties:

(1) $\alpha(S)=0$ if and only if $S$ is a relatively compact set.

(2) $S \subset T$ implies that $\alpha(S) \leq \alpha(T)$.

(3) $\alpha(\bar{S})=\alpha(S)$.

(4) $\alpha(S \bigcup T)=\max \{\alpha(S), \alpha(T)\}$.

(5) $\alpha(a S)=|a| \alpha(S)$.

(6) $\alpha(S+T) \leq \alpha(S)+\alpha(T)$.

(7) $\alpha(\overline{c o} S)=\alpha(S)$, where $\overline{c o} S$ is the convex closure of $S$.

(8) $|\alpha(S)-\alpha(T)| \leq 2 d_{h}(S, T)$, where $d_{h}(S, T)$ denotes the Hausdorff distance between the sets $S$ and $T$, that is,

$$
d_{h}(S, T)=\max \left\{\sup _{x \in S} d(x, T), \sup _{x \in T} d(x, S)\right\}
$$

where $d(\cdot, \cdot)$ denotes the distance from an element of $E$ to a set of $E$.

Lemma 2.2 ([42]) Let $E$ be a Banach space, and let $D \subset E$ be bounded. Then there exists a countable set $D_{0} \subset D$, such that $\alpha(D) \leq 2 \alpha\left(D_{0}\right)$.

Lemma 2.3 ([43]) Let $E$ be a Banach space, and let $D \subset C(I, E)$ be equicontinuous and bounded, then $\alpha(D(t))$ is continuous on $I$, and $\alpha(D)=\max _{t \in I} \alpha(D(t))$.

Lemma 2.4 ([44]) Let E be a Banach space, and let $D=\left\{u_{n}\right\} \subset C(I, E)$ be a bounded and countable set. Then $\alpha(D(t))$ is the Lebesgue integral on e, and

$$
\alpha\left(\left\{\int_{I} u_{n}(t) d t \mid n \in \mathbb{N}\right\}\right) \leq 2 \int_{I} \alpha(D(t)) d t .
$$

Lemma 2.5 ([45]) Let $E$ be a Banach space. Assume that $D \subset E$ is a bounded closed and convex set on $E, Q: D \rightarrow D$ is condensing. Then $Q$ has at least one fixed point in $D$.

We recall the abstract degenerate Cauchy problem as follows [35]:

$$
\left\{\begin{array}{l}
\frac{d}{d t} B u(t)=A u(t), \quad t \in J \\
B u(0)=B u_{0}
\end{array}\right.
$$


Definition 2.6 (See [19, Definition 1.4]) A strongly continuous operator family $\{W(t)\}_{t \geq 0}$ of $D(B)$ to a Banach space $E$, satisfying the requirement that $\{W(t)\}_{t \geq 0}$ is exponentially bounded, which means that for any $u \in D(B)$ there exist $a>0, M>0$ such that

$$
\|W(t) u\| \leq M e^{a t}\|u\|, \quad t \geq 0,
$$

is called an exponentially bounded propagation family for (2.1) if for $\lambda>a$,

$$
(\lambda B-A)^{-1} B u=\int_{0}^{\infty} e^{-\lambda t} W(t) u d t, \quad u \in D(B) .
$$

In this case, we also say that (2.1) has an exponentially bounded propagation family $\{W(t)\}_{t \geq 0}$.

Motivated by the above definition, we can give the following definition.

Definition 2.7 Let $A: D(A) \subseteq E \rightarrow E, B: D(B) \subseteq E \rightarrow E$ be closed linear operators defined on a Banach space $E$ satisfying $D(A) \cap D(B) \neq\{0\}$. Let $\mu>0$. We say the pair $(A, B)$ is the generator of an $\mu$-resolvent family, if there exist $a \geq 0$ and a strongly continuous function $K_{\mu}:[0, \infty) \rightarrow B(E)$ such that $K_{\mu}(t)$ is exponentially bounded, $\left\{\lambda^{\mu}: \operatorname{Re} \lambda>a\right\} \subset \rho(A)$, and for all $x \in E$,

$$
\left(\lambda^{\mu} B-A\right)^{-1} B u=\int_{0}^{\infty} e^{-\lambda t} K_{\mu}(t) u d t, \quad \operatorname{Re} \lambda>a .
$$

In this case, $\left\{K_{\mu}(t)\right\}_{t \geq 0}$ is called the $\mu$-resolvent family generated by the pair $(A, B)$.

Lemma 2.6 ([34]) Let $f: J \times E \times E \rightarrow E$ be a function such that $f \in C_{1-\gamma}(J)$ for any $u \in$ $C_{1-\gamma}(J)$. A function $u \in C_{1-\gamma}(J)$ is a solution of the fractional initial value problem

$$
\left\{\begin{array}{l}
D_{0+}^{\nu, \mu}(B u(t))=A u(t)+B f\left(t, u(t), \int_{0}^{t} \rho(t, s) h(t, s, u(s)) d s\right), \quad t \in J, \\
I_{0+}^{1-\gamma} B u(0)=B u_{0}
\end{array}\right.
$$

if $u$ satisfies

$$
u(t)=S_{v, \mu}(t) u_{0}+\int_{0}^{t} K_{\mu}(t-s) f\left(s, u(s), \int_{0}^{s} \rho(s, \tau) h(s, \tau, u(\tau)) d \tau\right) d s
$$

where

$$
S_{\nu, \mu}(t)=I_{0+}^{\nu(1-\mu)} K_{\mu}(t), \quad K_{\mu}(t)=t^{\mu-1} P_{\mu}(t), \quad P_{\mu}(t)=\mu \int_{0}^{\infty} \sigma \xi_{\mu}(\sigma) W\left(t^{\mu} \sigma\right) d \sigma,
$$

the function $\xi_{\mu}$ is a probability density function defined on $(0, \infty)$ such that

$$
\xi_{\mu}(\sigma)=\frac{1}{\mu} \sigma^{-1-\frac{1}{\mu}} \varpi_{\mu}\left(\sigma^{-\frac{1}{\mu}}\right) \geq 0
$$

and the one sided stable probability density in [24] is as follows:

$$
\varpi_{\mu}(\sigma)=\frac{1}{\pi} \sum_{n=1}^{\infty}(-1)^{n-1} \sigma^{-\mu n-1} \frac{\Gamma(n \mu+1)}{n !} \sin (n \pi \mu), \quad \sigma \in(0, \infty) .
$$


Lemma 2.7 Suppose $A$ and $B$ are closed (unbounded) linear operator with domains contained in $E, 0 \leq v \leq 1,0<\mu<1$, then

$$
D_{0+}^{v, \mu}\left[B S_{v, \mu}(t) u_{0}\right]=A\left[S_{v, \mu}(t) u_{0}\right]
$$

and

$$
\begin{aligned}
D_{0+}^{v, \mu} & \left(\int_{0}^{t} K_{\mu}(t-s) B f\left(s, u(s), \int_{0}^{s} \rho(s, \tau) h(s, \tau, u(\tau)) d \tau\right) d s\right) \\
= & A \int_{0}^{t} K_{\mu}(t-s) f\left(s, u(s), \int_{0}^{s} \rho(s, \tau) h(s, \tau, u(\tau)) d \tau\right) d s \\
& +B f\left(s, u(s), \int_{0}^{s} \rho(s, \tau) h(s, \tau, u(\tau)) d \tau\right) .
\end{aligned}
$$

Proof By Definition 2.7, let $\lambda>a$ be fixed, then we have

$$
\left(\lambda^{\mu} B-A\right)^{-1} B u=\int_{0}^{\infty} e^{-\lambda t} K_{\mu}(t) u d t, \quad u \in D(B) .
$$

It follows from (2.4) and the Laplace transform; it is obvious that

$$
\begin{aligned}
\mathcal{L}\left(B S_{\nu, \mu}(t) u_{0}\right) & =\mathcal{L}\left(I_{0+}^{\nu(1-\mu)} B K_{\mu}(t) u_{0}\right) \\
& =\lambda^{\nu(\mu-1)} B\left(\lambda^{\mu} B-A\right)^{-1} B u_{0} .
\end{aligned}
$$

The difference between fractional derivatives of different types becomes apparent from the Laplace transformation. In [33] it is found for $0<\mu<1$ that

$$
\mathcal{L}\left[D_{0+}^{\mu, v} f(x)\right](\lambda)=\lambda^{\mu} \mathcal{L}[f(x)](\lambda)-\lambda^{\nu(\mu-1)}\left(I_{0+}^{(1-v)(1-\mu)} f\right)(0+)
$$

where $\left(I_{0+}^{(1-v)(1-\mu)} f\right)(0+)$ is the Riemann-Liouville fractional integral of order $(1-v)(1-\mu)$ evaluated in the limits as $t \rightarrow 0+$, it being understood that

$$
\mathcal{L}[f(x)](\lambda)=\int_{0}^{\infty} e^{-\lambda x} f(x) d x
$$

Therefore, we obtain

$$
\begin{aligned}
\mathcal{L}\left(D_{0+}^{v, \mu}\left[B S_{v, \mu}(t) u_{0}\right]\right) & =\lambda^{\mu} \mathcal{L}\left(B S_{\nu, \mu}(t) u_{0}\right)-\lambda^{\nu(\mu-1)} B u_{0} \\
& =\lambda^{\mu} B\left[\lambda^{\nu(\mu-1)}\left(\lambda^{\mu} B-A\right)^{-1} B\right] u_{0}-\lambda^{\nu(\mu-1)} B u_{0} \\
& =\lambda^{\nu(\mu-1)}\left(\lambda^{\mu} B-A\right)^{-1} B\left[\lambda^{\mu} B-\left(\lambda^{\mu} B-A\right)\right] u_{0} \\
& =\lambda^{\nu(\mu-1)}\left(\lambda^{\mu} B-A\right)^{-1} B\left[\lambda^{\mu} B-\lambda^{\mu} B+A\right] u_{0} \\
& =\lambda^{\nu(\mu-1)}\left(\lambda^{\mu} B-A\right)^{-1} B A u_{0} \\
& =A \lambda^{\nu(\mu-1)}\left(\lambda^{\mu} B-A\right)^{-1} B u_{0} .
\end{aligned}
$$


Combining (2.4) and (2.6) yields

$$
D_{0+}^{v, \mu}\left[B S_{v, \mu}(t) u_{0}\right]=A\left[S_{v, \mu}(t) u_{0}\right]
$$

Similarly, we have

$$
\begin{array}{r}
\mathcal{L}\left(\int_{0}^{t} K_{\mu}(t-s) B f\left(s, u(s), \int_{0}^{s} \rho(s, \tau) h(s, \tau, u(\tau)) d \tau\right) d s\right) \\
\quad=\mathcal{L}\left(K_{\mu}(t)\right) \cdot \mathcal{L}\left(B f\left(s, u(s), \int_{0}^{s} \rho(s, \tau) h(s, \tau, u(\tau)) d \tau\right)\right)
\end{array}
$$

and

$$
\begin{aligned}
\mathcal{L}( & \left.D_{0+}^{v, \mu}\left[\int_{0}^{t} K_{\mu}(t-s) B f\left(s, u(s), \int_{0}^{s} \rho(s, \tau) h(s, \tau, u(\tau)) d \tau\right) d s\right]\right) \\
= & \lambda^{\mu} \mathcal{L}\left(\int_{0}^{t} K_{\mu}(t-s) B f\left(s, u(s), \int_{0}^{s} \rho(s, \tau) h(s, \tau, u(\tau)) d \tau\right) d s\right)-\lambda^{\nu(\mu-1)} \cdot 0 \\
= & \lambda^{\mu} \mathcal{L}\left(K_{\mu}(t)\right) \cdot \mathcal{L}\left(B f\left(s, u(s), \int_{0}^{s} \rho(s, \tau) h(s, \tau, u(\tau)) d \tau\right)\right) \\
= & \lambda^{\mu}\left(\lambda^{\mu} B-A\right)^{-1} B \cdot \mathcal{L}\left(B f\left(s, u(s), \int_{0}^{s} \rho(s, \tau) h(s, \tau, u(\tau)) d \tau\right)\right) \\
= & \left(\lambda^{\mu} B-A+A\right)\left(\lambda^{\mu} B-A\right)^{-1} B \cdot \mathcal{L}\left(f\left(s, u(s), \int_{0}^{s} \rho(s, \tau) h(s, \tau, u(\tau)) d \tau\right)\right) \\
= & A\left(\lambda^{\mu} B-A\right)^{-1} B \cdot \mathcal{L}\left(f\left(s, u(s), \int_{0}^{s} \rho(s, \tau) h(s, \tau, u(\tau)) d \tau\right)\right) \\
& +B \cdot \mathcal{L}\left(f\left(s, u(s), \int_{0}^{s} \rho(s, \tau) h(s, \tau, u(\tau)) d \tau\right)\right) .
\end{aligned}
$$

Thus, it follows from (2.7) and (2.8) that

$$
\begin{aligned}
D_{0+}^{v, \mu} & {\left[\int_{0}^{t} K_{\mu}(t-s) B f\left(s, u(s), \int_{0}^{s} \rho(s, \tau) h(s, \tau, u(\tau)) d \tau\right) d s\right] } \\
= & A \int_{0}^{t} K_{\mu}(t-s) f\left(s, u(s), \int_{0}^{s} \rho(s, \tau) h(s, \tau, u(\tau)) d \tau\right) d s \\
& +B f\left(s, u(s), \int_{0}^{s} \rho(s, \tau) h(s, \tau, u(\tau)) d \tau\right) .
\end{aligned}
$$

According to the above fundamental result, a new and important equivalent mixed type integral equation for the problem can be established.

Lemma 2.8 Let $f$ be a functions such that $f(\cdot, v, w) \in C_{1-\gamma}(J)$ for any $u \in C_{1-\gamma}(J)$. A function $u \in C_{1-\gamma}^{\gamma}(J)$ is a solution of $E q$. (1.1) if and only if $u$ satisfies the mixed type integral equation

$$
\begin{aligned}
u(t)= & S_{v, \mu}(t) d \sum_{i=1}^{m} \lambda_{i} I_{0+}^{\gamma} \int_{0}^{\tau_{i}} K_{\mu}\left(\tau_{i}-s\right) f\left(s, u(s), \int_{0}^{s} \rho(s, \tau) h(s, \tau, u(\tau)) d \tau\right) d s \\
& +\int_{0}^{t} K_{\mu}(t-s) f\left(s, u(s), \int_{0}^{s} \rho(s, \tau) h(s, \tau, u(\tau)) d \tau\right) d s,
\end{aligned}
$$


where

$$
d=\frac{1}{1-\sum_{i=1}^{m} \lambda_{i} I_{0+}^{\gamma} S_{v, \mu}\left(\tau_{i}\right)}
$$

Proof According to Lemma 2.6, a solution of Eq. (1.1) can be expressed by

$$
u(t)=S_{v, \mu}(t) I_{0+}^{1-\gamma} u(0)+\int_{0}^{t} K_{\mu}(t-s) f\left(s, u(s), \int_{0}^{s} \rho(s, \tau) h(s, \tau, u(\tau)) d \tau\right) d s .
$$

Next, we substitute $t=\tau_{i}$ into the above equation, we have

$$
\begin{aligned}
\lambda_{i} u\left(\tau_{i}\right)= & \lambda_{i} S_{v, \mu}\left(\tau_{i}\right) u_{0} \\
& +\lambda_{i} \int_{0}^{\tau_{i}} K_{\mu}\left(\tau_{i}-s\right) f\left(s, u(s), \int_{0}^{s} \rho(s, \tau) h(s, \tau, u(\tau)) d \tau\right) d s .
\end{aligned}
$$

Thus, we have

$$
\begin{aligned}
I_{0+}^{1-\gamma} B u(0)= & \sum_{i=1}^{m} \lambda_{i} I_{0+}^{\gamma} B u\left(\tau_{i}\right) \\
= & \sum_{i=1}^{m} I_{0+}^{\gamma} \lambda_{i} B u\left(\tau_{i}\right) \\
= & \sum_{i=1}^{m} \lambda_{i} I_{0+}^{\gamma} S_{v, \mu}\left(\tau_{i}\right) B u_{0} \\
& +\sum_{i=1}^{m} \lambda_{i} I_{0+}^{\gamma} \int_{0}^{\tau_{i}} K_{\mu}\left(\tau_{i}-s\right) B f\left(s, u(s), \int_{0}^{s} \rho(s, \tau) h(s, \tau, u(\tau)) d \tau\right) d s \\
= & I_{0+}^{1-\gamma} B u(0) \sum_{i=1}^{m} \lambda_{i} I_{0+}^{\gamma} S_{v, \mu}\left(\tau_{i}\right) \\
& +\sum_{i=1}^{m} \lambda_{i} I_{0+}^{\gamma} \int_{0}^{\tau_{i}} K_{\mu}\left(\tau_{i}-s\right) B f\left(s, u(s), \int_{0}^{s} \rho(s, \tau) h(s, \tau, u(\tau)) d \tau\right) d s,
\end{aligned}
$$

which implies

$$
I_{0+}^{1-\gamma} B u(0)=d \sum_{i=1}^{m} \lambda_{i} I_{0+}^{\gamma} \int_{0}^{\tau_{i}} K_{\mu}\left(\tau_{i}-s\right) B f\left(s, u(s), \int_{0}^{s} \rho(s, \tau) h(s, \tau, u(\tau)) d \tau\right) d s
$$

i.e.

$$
I_{0+}^{1-\gamma} u(0)=u_{0}=d \sum_{i=1}^{m} \lambda_{i} I_{0+}^{\gamma} \int_{0}^{\tau_{i}} K_{\mu}\left(\tau_{i}-s\right) f\left(s, u(s), \int_{0}^{s} \rho(s, \tau) h(s, \tau, u(\tau)) d \tau\right) d s
$$

Submitting (2.12) to (2.10), we derive that (2.9). It is probative that $u$ is also a solution of the integral of Eq. (2.2) when $u$ is a solution of Eq. (1.1). 
The necessity has been already proved, next, we are read to prove its sufficiency. Applying $I_{0+}^{1-\gamma}$ to both sides of (2.9), we have

$$
\begin{aligned}
& I_{0+}^{1-\gamma} B u(t) \\
& =I_{0+}^{1-\gamma} S_{v, \mu}(t) d \sum_{i=1}^{m} \lambda_{i} I_{0+}^{\gamma} \int_{0}^{\tau_{i}} K_{\mu}\left(\tau_{i}-s\right) B f\left(s, u(s), \int_{0}^{s} \rho(s, \tau) h(s, \tau, u(\tau)) d \tau\right) d s \\
& \quad+I_{0+}^{1-\gamma} \int_{0}^{t} K_{\mu}(t-s) B f\left(s, u(s), \int_{0}^{s} \rho(s, \tau) h(s, \tau, u(\tau)) d \tau\right) d s .
\end{aligned}
$$

Substituting $t=\tau_{i}$ into (2.9), we have

$$
\begin{aligned}
u\left(\tau_{i}\right)= & S_{v, \mu}\left(\tau_{i}\right) d \sum_{i=1}^{m} \lambda_{i} I_{0+}^{\gamma} \int_{0}^{\tau_{i}} K_{\mu}\left(\tau_{i}-s\right) f\left(s, u(s), \int_{0}^{s} \rho(s, \tau) h(s, \tau, u(\tau)) d \tau\right) d s \\
& +\int_{0}^{\tau_{i}} K_{\mu}\left(\tau_{i}-s\right) f\left(s, u(s), \int_{0}^{s} \rho(s, \tau) h(s, \tau, u(\tau)) d \tau\right) d s
\end{aligned}
$$

Then we derive

$$
\begin{aligned}
& \sum_{i=1}^{m} \lambda_{i} I_{0+}^{\gamma} B u\left(\tau_{i}\right) \\
& =\sum_{i=1}^{m} \lambda_{i} I_{0+}^{\gamma} S_{v, \mu}\left(\tau_{i}\right) d \sum_{i=1}^{m} \lambda_{i} I_{0+}^{\gamma} \int_{0}^{\tau_{i}} K_{\mu}\left(\tau_{i}-s\right) B f\left(s, u(s), \int_{0}^{s} \rho(s, \tau) h(s, \tau, u(\tau)) d \tau\right) d s \\
& +\sum_{i=1}^{m} \lambda_{i} I_{0+}^{\gamma} \int_{0}^{\tau_{i}} K_{\mu}\left(\tau_{i}-s\right) B f\left(s, u(s), \int_{0}^{s} \rho(s, \tau) h(s, \tau, u(\tau)) d \tau\right) d s \\
& =\frac{\sum_{i=1}^{m} \lambda_{i} I_{0+}^{\gamma} S_{v, \mu}\left(\tau_{i}\right)}{1-\sum_{i=1}^{m} \lambda_{i} I_{0+}^{\gamma} S_{v, \mu}\left(\tau_{i}\right)} \\
& \times \sum_{i=1}^{m} \lambda_{i} I_{0+}^{\gamma} \int_{0}^{\tau_{i}} K_{\mu}\left(\tau_{i}-s\right) B f\left(s, u(s), \int_{0}^{s} \rho(s, \tau) h(s, \tau, u(\tau)) d \tau\right) d s \\
& +\sum_{i=1}^{m} \lambda_{i} I_{0+}^{\gamma} \int_{0}^{\tau_{i}} K_{\mu}\left(\tau_{i}-s\right) B f\left(s, u(s), \int_{0}^{s} \rho(s, \tau) h(s, \tau, u(\tau)) d \tau\right) d s \\
& =(d-1) \sum_{i=1}^{m} \lambda_{i} I_{0+}^{\gamma} \int_{0}^{\tau_{i}} K_{\mu}\left(\tau_{i}-s\right) B f\left(s, u(s), \int_{0}^{s} \rho(s, \tau) h(s, \tau, u(\tau)) d \tau\right) d s \\
& +\sum_{i=1}^{m} \lambda_{i} I_{0+}^{\gamma} \int_{0}^{\tau_{i}} K_{\mu}\left(\tau_{i}-s\right) B f\left(s, u(s), \int_{0}^{s} \rho(s, \tau) h(s, \tau, u(\tau)) d \tau\right) d s \\
& =d \sum_{i=1}^{m} \lambda_{i} I_{0+}^{\gamma} \int_{0}^{\tau_{i}} K_{\mu}\left(\tau_{i}-s\right) B f\left(s, u(s), \int_{0}^{s} \rho(s, \tau) h(s, \tau, u(\tau)) d \tau\right) d s .
\end{aligned}
$$

It follows (2.12) and (2.13) that

$$
I_{0+}^{1-\gamma} B u(0)=\sum_{i=1}^{m} \lambda_{i} I_{0+}^{1-\gamma} B u\left(\tau_{i}\right) .
$$


Next, by applying $D_{0+}^{\nu, \mu}$ to both sides of (2.9) and using Lemma 2.7, we can obtain

$$
\begin{aligned}
& D_{0+}^{\nu, \mu}(B u)=D_{0+}^{\nu, \mu}\left[B S_{v, \mu}(t) d \sum_{i=1}^{m} \lambda_{i} I_{0+}^{\gamma}\right. \\
& \times \int_{0}^{\tau_{i}} K_{\mu}\left(\tau_{i}-s\right) f\left(s, u(s), \int_{0}^{s} \rho(s, \tau) h(s, \tau, u(\tau)) d \tau\right) d s \\
& \left.+\int_{0}^{t} K_{\mu}(t-s) B f\left(s, u(s), \int_{0}^{s} \rho(s, \tau) h(s, \tau, u(\tau)) d \tau\right) d s\right] \\
& =D_{0+}^{v, \mu}\left[B S_{\nu, \mu}(t) d \sum_{i=1}^{m} \lambda_{i} I_{0+}^{\gamma}\right. \\
& \left.\times \int_{0}^{\tau_{i}} K_{\mu}\left(\tau_{i}-s\right) f\left(s, u(s), \int_{0}^{s} \rho(s, \tau) h(s, \tau, u(\tau)) d \tau\right) d s\right] \\
& +D_{0+}^{v, \mu}\left[\int_{0}^{t} K_{\mu}(t-s) B f\left(s, u(s), \int_{0}^{s} \rho(s, \tau) h(s, \tau, u(\tau)) d \tau\right) d s\right] \\
& =\left[d \sum_{i=1}^{m} \lambda_{i} I_{0+}^{\gamma} \int_{0}^{\tau_{i}} K_{\mu}\left(\tau_{i}-s\right) f\left(s, u(s), \int_{0}^{s} \rho(s, \tau) h(s, \tau, u(\tau)) d \tau\right) d s\right] \\
& \times D_{0+}^{v, \mu}\left[B S_{v, \mu}(t)\right] \\
& +D_{0+}^{v, \mu}\left[\int_{0}^{t} K_{\mu}(t-s) B f\left(s, u(s), \int_{0}^{s} \rho(s, \tau) h(s, \tau, u(\tau)) d \tau\right) d s\right] \\
& =\left[d \sum_{i=1}^{m} \lambda_{i} I_{0+}^{\gamma} \int_{0}^{\tau_{i}} K_{\mu}\left(\tau_{i}-s\right) f\left(s, u(s), \int_{0}^{s} \rho(s, \tau) h(s, \tau, u(\tau)) d \tau\right) d s\right] A S_{\nu, \mu}(t) \\
& +A \int_{0}^{t} K_{\mu}(t-s) f\left(s, u(s), \int_{0}^{s} \rho(s, \tau) h(s, \tau, u(\tau)) d \tau\right) d s \\
& +B f\left(s, u(s), \int_{0}^{s} \rho(s, \tau) h(s, \tau, u(\tau)) d \tau\right) \\
& =A\left(S_{v, \mu}(t) d \sum_{i=1}^{m} \lambda_{i} I_{0+}^{\gamma} \int_{0}^{\tau_{i}} K_{\mu}\left(\tau_{i}-s\right) f\left(s, u(s), \int_{0}^{s} \rho(s, \tau) h(s, \tau, u(\tau)) d \tau\right) d s\right. \\
& \left.+\int_{0}^{t} K_{\mu}(t-s) f\left(s, u(s), \int_{0}^{s} \rho(s, \tau) h(s, \tau, u(\tau)) d \tau\right) d s\right) \\
& +B f\left(s, u(s), \int_{0}^{s} \rho(s, \tau) h(s, \tau, u(\tau)) d \tau\right) \\
& =A u(t)+B f\left(s, u(s), \int_{0}^{s} \rho(s, \tau) h(s, \tau, u(\tau)) d \tau\right) .
\end{aligned}
$$

Hence, it reduces to $D_{0+}^{v, \mu}(B u)=A u(t)+B f\left(s, u(s), \int_{0}^{s} \rho(s, \tau) h(s, \tau, u(\tau)) d \tau\right)$. The results are proved completely.

Lemma 2.9 (See [46, Property 2.1]) If $\alpha>$ and $\beta>0$, then

$$
\left[I_{a+}^{\alpha}(t-a)^{\beta-1}\right](x)=\frac{\Gamma(\beta)}{\Gamma(\alpha+\beta)}(x-a)^{\alpha+\beta-1} .
$$


To end this section, we give the following lemma.

Lemma 2.10 Let $\mu>0, k>0, z \in R$ and $f \in C(J)$, then

$$
I_{0+}^{k} \int_{0}^{z}(z-t)^{\mu-1} P_{\mu}(t-s) f(s) d s=\frac{B(\mu, k)}{\Gamma(k)} \int_{0}^{z}(z-t)^{\mu+k-1} P_{\mu}(t-s) f(s) d s .
$$

Proof According to Lemma 2.9, we have

$$
\begin{aligned}
I_{0+}^{k} & \int_{0}^{z}(z-t)^{\mu-1} P_{\mu}(t-s) f(s) d s \\
= & \frac{1}{\Gamma(k)} \int_{0}^{z}(z-u)^{k-1} \int_{0}^{u}(u-t)^{\mu-1} P_{\mu}(t-s) f(s) d s d u \\
& =\frac{1}{\Gamma(k)} \int_{0}^{z} \int_{t}^{z}(z-u)^{k-1}(u-t)^{\mu-1} P_{\mu}(t-s) f(s) d s d u \\
& =\frac{1}{\Gamma(k)} \int_{0}^{z} P_{\mu}(t-s) f(s) d s \int_{t}^{z}(z-u)^{k-1}(u-t)^{\mu-1} d u \\
& =\frac{1}{\Gamma(k)} \int_{0}^{z} B(\mu, k)(z-t)^{\mu+k-1} P_{\mu}(t-s) f(s) d s \\
& =\frac{B(\mu, k)}{\Gamma(k)} \int_{0}^{z}(z-t)^{\mu+k-1} P_{\mu}(t-s) f(s) d s .
\end{aligned}
$$

The desired result is obtained.

Lemma 2.11 ([34]) Assume that $\{W(t)\}_{t \geq 0}$ is a norm continuous family for $t>0$ and $\|W(t)\| \leq M$, for any fixed $t>0,\left\{K_{\mu}(t)\right\}_{t>0}$, and $\left\{S_{\nu, \mu}(t)\right\}_{t>0}$ are linear operators, and for any $u \in E$

$$
\left\|K_{\mu}(t)\right\| \leq \frac{M t^{\mu-1}}{\Gamma(\mu)}, \quad\left\|S_{v, \mu}(t)\right\| \leq \frac{M t^{(\nu-1)(1-\mu)}}{\Gamma(v(1-\mu)+\mu)} .
$$

Lemma 2.12 ([34]) Assume that $\{W(t)\}_{t \geq 0}$ is a norm continuous family for $t>0$ and $\|W(t)\| \leq M,\left\{K_{\mu}(t)\right\}_{t>0}$ and $\left\{S_{v, \mu}(t)\right\}_{t>0}$ are strongly continuous for $t>0$.

\section{Main results}

In this section, we will state and prove our main results. First of all, we introduce the following assumptions:

(H1) $\{W(t)\}_{t \geq 0}$ is a norm continuous family for $t>0$ and uniformly bounded, i.e., there exists $M \geq 1$ such that $\|W(t)\| \leq M$.

(H2) (i) $f: J \times E \times E \rightarrow D(B) \subset E$ with $f(\cdot, v, w): J \rightarrow D(B) \subset E$ is measurable for all $(v, w) \in E \times E$ and $f(t, \cdot, \cdot): E \times E \rightarrow D(B) \subset E$ is continuous for a.e. $t \in J$, and there exist a function $\mu_{1} \in L_{\frac{1}{q}}\left(J, R^{+}\right)\left(\frac{1}{q}>1\right)$ and a continuous function $\mu_{2}(\cdot)$ such that

$$
\| f\left(t, v, w\left\|\leq \mu_{1}(t)\right\| v\left\|+\mu_{2}(t)\right\| w \|\right.
$$

and

$$
I_{0+}^{\mu} \mu_{1} \in C\left(J^{\prime}, R^{+}\right), \quad \lim _{t \rightarrow 0+} t^{1-\gamma} I_{0+}^{\mu} \mu_{1}(t)=0,
$$




$$
I_{0+}^{\mu} \mu_{2} \in C\left(J^{\prime}, R^{+}\right), \quad \lim _{t \rightarrow 0+} t^{1-\gamma} I_{0+}^{\mu} \mu_{2}(t)=0
$$

for almost all $t \in J$.

(ii) There exist functions $\eta(\cdot) \in L_{\frac{1}{q}}\left(J, R^{+}\right), \xi(\cdot) \in L_{\frac{1}{q}}\left(J, R^{+}\right)$and constants $L_{1}, L_{2}$ such that, for any bounded, equicontinuous and countable sets $D_{k} \subset E(k=1,2)$,

$$
\alpha\left(f\left(t, D_{1}, D_{2}\right)\right) \leq L_{1} \eta(t) \alpha\left(D_{1}\right)+L_{2} \xi(t) \alpha\left(D_{2}\right), \quad t \in J .
$$

(H3) (i) The function $h(t, s, \cdot): E \rightarrow E$ is continuous for a.e. $(t, s) \in \Delta$ for each $u \in E$, the function $h(\cdot, \cdot, u): \Delta \rightarrow E$ is measurable. Moreover, there exists a function $m: \Delta \rightarrow$ $R$ with $\sup _{t \in J} \int_{0}^{t} m(t, s) d s: m^{*}<\infty$ such that

$$
\|h(t, s, u)\| \leq m(t, s)\|u\|, \quad u \in E
$$

(ii) For any bounded set $D_{1} \in E$, and $0 \leq s \leq t \leq b$, there exists a function, a constant $\zeta: \Delta \rightarrow R$ such that

$$
\alpha\left(h\left(t, s, D_{1}\right)\right) \leq \zeta(t, s) \alpha\left(D_{1}\right)
$$

where $\sup _{t \in J} \int_{0}^{t} \zeta(t, s) d s:=\zeta^{*}<\infty$

(H4) For each $t \in J, \rho(t, \cdot)$ is measurable on $J$ and $\rho(t)=\sup \{|\rho(t, s)|, 0 \leq s \leq t\}$ is bounded on $J$. The map $t \rightarrow \rho_{t}$ is continuous from $J$ to $L^{\infty}(J, R)$, here, $\rho_{t}(s)=\rho(t, s)$.

Now we are ready to establish the first existence results for Eq. (1.1) by using the fixed point theorem.

Theorem 3.1 Assume (H1)-(H4) are satisfied. Then Eq. (1.1) has at least one mild solution in $C_{1-\gamma}^{\gamma}(J, E)$ provided that

$$
\begin{aligned}
& \frac{M^{2}|d|}{\Gamma(\gamma) \Gamma(\mu+\gamma)} \sum_{i=1}^{m}\left|\lambda_{i}\right|\left[\left(\frac{1-q}{\mu+\gamma-q}\right)^{1-q} \tau_{i}^{\mu+\gamma-q}\left\|\mu_{1}\right\|_{L_{\frac{1}{q}}[0, b]}+\frac{b^{\mu+\gamma} \varrho m^{*} \mu_{2}^{*}}{\mu+\gamma}\right] \\
& +\frac{M b^{1-\gamma}}{\Gamma(\mu)}\left[\left(\frac{1-q}{\mu-q}\right)^{1-q} b^{\mu-q}\left\|\mu_{1}\right\|_{L_{\frac{1}{q}}[0, b]}+\frac{b^{\mu} \varrho m^{*} \mu_{2}^{*}}{\mu}\right]<1
\end{aligned}
$$

and

$$
\frac{M b^{\mu-q}}{\Gamma(\mu)}\left(\frac{1-q}{\mu-q}\right)^{1-q}\left(L_{1}\|\eta\|_{L_{\frac{1}{q}}[0, b]}+L_{2} \varrho \zeta^{*}\|\xi\|_{L_{\frac{1}{q}}[0, b]}\right)<\frac{1}{4}
$$

Proof According to Lemma 2.8, it is sufficient to prove the existence result for the mixed type integral Eq. (2.9). Consider the operator $Q: C_{1-\gamma}(J, E) \rightarrow C_{1-\gamma}(J, E)$ defined by

$$
\begin{aligned}
(Q u)(t)= & S_{\nu, \mu}(t) d \sum_{i=1}^{m} \lambda_{i} I_{0+}^{\gamma} \int_{0}^{\tau_{i}} K_{\mu}\left(\tau_{i}-s\right) f\left(s, u(s), \int_{0}^{s} \rho(s, \tau) h(s, \tau, u(\tau)) d \tau\right) d s \\
& +\int_{0}^{t} K_{\mu}(t-s) f\left(s, u(s), \int_{0}^{s} \rho(s, \tau) h(s, \tau, u(\tau)) d \tau\right) d s .
\end{aligned}
$$


By direct calculation, we know that the operator $Q$ is well defined. From Lemma 2.8, it is easy to verify that the mild solution of problem (1.1) is equivalent to the fixed point of the operator $Q$ defined by (3.2). In the following, we will prove that the operator $Q$ : $C_{1-\gamma}(J, E) \rightarrow C_{1-\gamma}(J, E)$ has at least one fixed point by applying the fixed point theorem. Our proof will be divided into four steps.

Step 1. We firstly prove that the operator Q defined by (3.2) maps the bounded closed convex set $B_{r}:=\left\{u \in C_{1-\gamma}(J, E):\|u(t)\|_{C_{1-\gamma}} \leq r, t \in J\right\}$ into itself. Observe that

$$
\begin{aligned}
& \lim _{t \rightarrow 0+} t^{1-\gamma} S_{v, \mu}(t) u_{0} \\
& \quad=\lim _{t \rightarrow 0+} \frac{t^{1-\gamma}}{\Gamma(v(1-\mu))} \int_{0}^{t}(t-s)^{v(1-\mu)-1} K_{\mu}(s) u_{0} d s \\
& =\lim _{t \rightarrow 0+} \frac{1}{\Gamma(v(1-\mu))} \int_{0}^{1}(1-s)^{v(1-\mu)-1} K_{\mu}(s) u_{0} d s \\
& =\lim _{t \rightarrow 0+} \frac{1}{\Gamma(v(1-\mu)) \Gamma(\mu)} \int_{0}^{1}(1-s)^{v(1-\mu)-1} s^{\mu-1} u_{0} d s \\
& =\frac{u_{0}}{\Gamma(v(1-\mu)+\mu)} .
\end{aligned}
$$

Define $t^{1-\gamma}(Q u)(t)$ as follows:

$$
t^{1-\gamma}(Q u)(t):= \begin{cases}t^{1-\gamma} S_{\nu, \mu}(t) u_{0} & \\ +t^{1-\gamma} \int_{0}^{t} K_{\mu}(t-s) f\left(s, u(s), \int_{0}^{s} \rho(s, \tau) h(s, \tau, u(\tau)) d \tau\right) d s, & t \in J^{\prime} \\ \frac{u_{0}}{\Gamma(\nu(1-\mu)+\mu)}, & t=0 .\end{cases}
$$

Suppose this is not true. Then, for each $r>0$, there exists $u_{r}(\cdot) \in B_{r}$ for some $t \in J$ such that $\left\|\left(Q u_{r}\right)(t)\right\|>r$. Combining with Lemma 2.11, Lemma 2.12, the assumption (H1), (H2) and the Hölder inequality, we get

$$
\begin{aligned}
r<\| t^{1-\gamma} & \left(Q u_{r}\right)(t) \| \\
\leq & \left\|t^{1-\gamma} S_{v, \mu}(t) d \sum_{i=1}^{m} \lambda_{i} I_{0+}^{\gamma} \int_{0}^{\tau_{i}} K_{\mu}\left(\tau_{i}-s\right) f\left(s, u(s), \int_{0}^{s} \rho(s, \tau) h(s, \tau, u(\tau)) d \tau\right) d s\right\| \\
& +\left\|t^{1-\gamma} \int_{0}^{t} K_{\mu}(t-s) f\left(s, u(s), \int_{0}^{s} \rho(s, \tau) h(s, \tau, u(\tau)) d \tau\right) d s\right\| \\
\leq & \frac{M|d| B(\mu, \gamma)}{\Gamma(\gamma) \Gamma(\gamma)} \sum_{i=1}^{m} \\
& \times\left|\lambda_{i}\right| \int_{0}^{\tau_{i}}\left(\tau_{i}-s\right)^{\mu+\gamma-1} P_{\mu}\left(\tau_{i}-s\right)\left|f\left(s, u(s), \int_{0}^{s} \rho(s, \tau) h(s, \tau, u(\tau)) d \tau\right)\right| d s \\
& +\frac{M t^{1-\gamma}}{\Gamma(\mu)} \int_{0}^{t}(t-s)^{\mu-1}\left|f\left(s, u(s), \int_{0}^{s} \rho(s, \tau) h(s, \tau, u(\tau)) d \tau\right)\right| d s \\
\leq & \frac{M^{2}|d|}{\Gamma(\gamma) \Gamma(\mu+\gamma)} \\
& \times \sum_{i=1}^{m}\left|\lambda_{i}\right| \int_{0}^{\tau_{i}}\left(\tau_{i}-s\right)^{\mu+\gamma-1}\left[\mu(s) r+\mu(s) \varrho r \int_{0}^{s} m(s, \tau) d \tau\right] d s
\end{aligned}
$$




$$
\begin{aligned}
& +\frac{M t^{1-\gamma}}{\Gamma(\mu)} \int_{0}^{t}(t-s)^{\mu-1}\left[\mu_{1}(s) r+\mu_{2}(s) \varrho r \int_{0}^{s} m(s, \tau) d \tau\right] d s \\
\leq & \frac{M^{2}|d| r}{\Gamma(\gamma) \Gamma(\mu+\gamma)} \sum_{i=1}^{m}\left|\lambda_{i}\right|\left[\int_{0}^{\tau_{i}}\left(\tau_{i}-s\right)^{\mu+\gamma-1} \mu_{1}(s) d s+\frac{b^{\mu+\gamma} \varrho m^{*} \mu_{2}^{*}}{\mu+\gamma}\right] \\
& +\frac{r M t^{1-\gamma}}{\Gamma(\mu)}\left[\int_{0}^{t}(t-s)^{\mu-1} \mu_{1}(s) d s+\frac{b^{\mu} \varrho m^{*} \mu_{2}^{*}}{\mu}\right] \\
\leq & \frac{M^{2}|d| r}{\Gamma(\gamma) \Gamma(\mu+\gamma)} \sum_{i=1}^{m}\left|\lambda_{i}\right|\left[\left(\frac{1-q}{\mu+\gamma-q}\right)^{1-q} \tau_{i}^{\mu+\gamma-q}\left\|\mu_{1}\right\|_{L_{\frac{1}{q}}[0, b]}+\frac{b^{\mu+\gamma} \varrho m^{*} \mu_{2}^{*}}{\mu+\gamma}\right] \\
& +\frac{r M b^{1-\gamma}}{\Gamma(\mu)}\left[\left(\frac{1-q}{\mu-q}\right)^{1-q} b^{\mu-q}\left\|\mu_{1}\right\|_{L_{\frac{1}{q}}[0, b]}+\frac{b^{\mu} \varrho m^{*} \mu_{2}^{*}}{\mu}\right] .
\end{aligned}
$$

Dividing both sides of (3.3) by $r$, and taking the lower limit as $r \rightarrow+\infty$, combined with (3.1), we get

$$
\begin{aligned}
1 \leq & \frac{M^{2}|d|}{\Gamma(\gamma) \Gamma(\mu+\gamma)} \sum_{i=1}^{m}\left|\lambda_{i}\right|\left[\left(\frac{1-q}{\mu+\gamma-q}\right)^{1-q} \tau_{i}^{\mu+\gamma-q}\left\|\mu_{1}\right\|_{L_{\frac{1}{q}}[0, b]}+\frac{b^{\mu+\gamma} \varrho m^{*} \mu_{2}^{*}}{\mu+\gamma}\right] \\
& +\frac{M b^{1-\gamma}}{\Gamma(\mu)}\left[\left(\frac{1-q}{\mu-q}\right)^{1-q} b^{\mu-q}\left\|\mu_{1}\right\|_{L_{\frac{1}{q}}[0, b]}+\frac{b^{\mu} \varrho m^{*} \mu_{2}^{*}}{\mu}\right]<1,
\end{aligned}
$$

which is a contradiction. Therefore for some $r>0, Q\left(B_{r}\right) \subset B_{r}$.

Step 2. Now we show that $Q$ is continuous from $B_{r}$ into $B_{r}$. To show this, for any $u_{n}, u \in$ $B_{r}, n=1,2, \ldots$, with $\lim _{n \rightarrow \infty}\left\|u_{n}-u\right\|_{C_{1-\gamma}}=0$, we get

$$
\lim _{n \rightarrow \infty} u_{n}(t)=u(t)
$$

for all $t \in J$. By (H2)(i) and (H3)(i), we see that, for almost every $t \in J$,

$$
f\left(t, u_{n}(t), \int_{0}^{t} \rho(t, s) h\left(t, s, u_{n}(s)\right) d s\right) \rightarrow f\left(t, u(t), \int_{0}^{t} \rho(t, s) h(t, s, u(s)) d s\right)
$$

as $n \rightarrow \infty$.

Thus,

$$
\begin{aligned}
\lim _{n \rightarrow \infty} \| f\left(t, u_{n}(t), \int_{0}^{t} \rho(t, s) h\left(t, s, u_{n}(s)\right) d s\right) \\
\quad-f\left(t, u(t), \int_{0}^{t} \rho(t, s) h(t, s, u(s)) d s\right) \|_{C_{1-\gamma}}=0 .
\end{aligned}
$$

Noting that $u_{n} \rightarrow u$ in $C_{1-\gamma}(J, E)$, we infer that there exists $\varepsilon>0$ such that $\left\|u_{n}-u\right\| \leq \varepsilon$ for $n$ sufficiently large. Therefore, we have

$$
\begin{aligned}
(t-s)^{\mu-1} \| f\left(t, u_{n}(t), \int_{0}^{t} \rho(t, s) h\left(t, s, u_{n}(s)\right) d s\right)-f\left(t, u(t), \int_{0}^{t} \rho(t, s) h(t, s, u(s)) d s \|\right. \\
\leq \mu_{1}(t)\left(\left\|u_{n}(t)\right\|+\|u(t)\|\right) \\
\quad+\mu_{2}(t)\left(\int_{0}^{t}\left\|\rho(t, s) h\left(t, s, u_{n}(s)\right)\right\| d s-\int_{0}^{t}\|\rho(t, s) h(t, s, u(s)) d s\|\right)
\end{aligned}
$$




$$
\begin{aligned}
& \leq \mu_{1}(t)\left(\left\|u_{n}(t)-u(t)\right\|+2\|u(t)\|\right)+\mu_{2}(t) \int_{0}^{t} m(t, s)\left(\left\|u_{n}(t)-u(t)\right\|+2\|u(t)\|\right) d s \\
& \leq 2(t-s)^{\mu-1}\left(\mu_{1}(t)+\mu_{2}^{*} \varrho m^{*}\right)\left(\varepsilon+2 \sup _{t \in J}\|u(t)\|\right) .
\end{aligned}
$$

It follows from the Lebesgue dominated convergence theorem that, for $t \in J, u_{n}, u \in B_{r}$, we have

$$
\begin{aligned}
& \left\|t^{1-\gamma}\left[\left(Q u_{n}\right)(t)-(Q u)(t)\right]\right\| \\
& \leq \| t^{1-\gamma} S_{v, \mu}(t) d \sum_{i=1}^{m} \lambda_{i} I_{0+}^{\gamma} \int_{0}^{\tau_{i}} K_{\mu}\left(\tau_{i}-s\right)\left[f\left(s, u_{n}(s), \int_{0}^{s} \rho(s, \tau) h\left(s, \tau, u_{n}(\tau)\right) d \tau\right)\right. \\
& \left.-f\left(s, u(s), \int_{0}^{s} \rho(s, \tau) h(s, \tau, u(\tau)) d \tau\right)\right] d s \\
& +t^{1-\gamma} \int_{0}^{t} K_{\mu}(t-s)\left[f\left(s, u_{n}(s), \int_{0}^{s} \rho(s, \tau) h\left(s, \tau, u_{n}(\tau)\right) d \tau\right)\right. \\
& \left.-f\left(s, u(s), \int_{0}^{s} \rho(s, \tau) h(s, \tau, u(\tau)) d \tau\right)\right] d s \| \\
& \leq \frac{M^{2}|d|}{\Gamma(\gamma) \Gamma(\mu+\gamma)} \sum_{i=1}^{m}\left|\lambda_{i}\right| \int_{0}^{\tau_{i}}\left(\tau_{i}-s\right)^{\mu+\gamma-1}\left[f\left(s, u_{n}(s), \int_{0}^{s} \rho(s, \tau) h\left(s, \tau, u_{n}(\tau)\right) d \tau\right)\right. \\
& \left.-f\left(s, u(s), \int_{0}^{s} \rho(s, \tau) h(s, \tau, u(\tau)) d \tau\right)\right] d s \\
& +\frac{M t^{1-\gamma}}{\Gamma(\mu)} \int_{0}^{t}(t-s)^{\mu-1}\left[f\left(s, u_{n}(s), \int_{0}^{s} \rho(s, \tau) h\left(s, \tau, u_{n}(\tau)\right) d \tau\right)\right. \\
& \left.-f\left(s, u(s), \int_{0}^{s} \rho(s, \tau) h(s, \tau, u(\tau)) d \tau\right)\right] d s \\
& \leq \frac{M^{2}|d| \cdot \tau_{i}^{\mu+2 \gamma-1}}{\Gamma(\mu+2 \gamma)} \sum_{i=1}^{m}\left|\lambda_{i}\right| \cdot \| f\left(s, u_{n}(s), \int_{0}^{s} \rho(s, \tau) h\left(s, \tau, u_{n}(\tau)\right) d \tau\right) \\
& -f\left(s, u(s), \int_{0}^{s} \rho(s, \tau) h(s, \tau, u(\tau)) d \tau\right) \|_{C_{1-\gamma}} \\
& +\frac{M b^{\mu} B(\mu, \gamma)}{\Gamma(\mu)} \| f\left(s, u_{n}(s), \int_{0}^{s} \rho(s, \tau) h\left(s, \tau, u_{n}(\tau)\right) d \tau\right) \\
& -f\left(s, u(s), \int_{0}^{s} \rho(s, \tau) h(s, \tau, u(\tau)) d \tau\right) \|_{C_{1-\gamma}} .
\end{aligned}
$$

This means

$$
\begin{aligned}
\left\|t^{1-\gamma}\left[\left(Q u_{n}\right)(t)-(Q u)(t)\right]\right\| \leq & \left(\frac{M^{2}|d| \cdot \tau_{i}^{\mu+2 \gamma-1}}{\Gamma(\mu+2 \gamma)} \sum_{i=1}^{m}\left|\lambda_{i}\right|+\frac{M b^{\mu} B(\mu, \gamma)}{\Gamma(\mu)}\right) \\
& \cdot \| f\left(s, u_{n}(s), \int_{0}^{s} \rho(s, \tau) h\left(s, \tau, u_{n}(\tau)\right) d \tau\right) \\
& -f\left(s, u(s), \int_{0}^{s} \rho(s, \tau) h(s, \tau, u(\tau)) d \tau\right) \|_{C_{1-\gamma}} .
\end{aligned}
$$


Therefore, we obtain

$$
\lim _{n \rightarrow \infty}\left\|Q u_{n}-Q u\right\|_{C_{1-\gamma}}=0
$$

this shows that $Q: B_{r} \rightarrow B_{r}$ is a continuous operator.

Step 3. We will prove that $\left\{Q u: u \in B_{r}\right\}$ is a family of equicontinuous function. For any $u \in B_{r}$ and $0 \leq t_{1}<t_{2} \leq b$, by (3.2) and the assumption (H1), (H2), we get

$$
\begin{aligned}
&\left\|t_{2}^{1-\gamma}(Q u)\left(t_{2}\right)-t_{1}^{1-\gamma}(Q u)\left(t_{1}\right)\right\| \\
& \leq\left\|t_{2}^{1-\gamma} S_{v, \mu}\left(t_{2}\right)-t_{1}^{1-\gamma} S_{v, \mu}\left(t_{1}\right)\right\| \\
& \quad \times\left\|d \sum_{i=1}^{m} \lambda_{i} I_{0+}^{\gamma} \int_{0}^{\tau_{i}} K_{\mu}\left(\tau_{i}-s\right) f\left(s, u(s), \int_{0}^{s} \rho(s, \tau) h(s, \tau, u(\tau)) d \tau\right) d s\right\| \\
&+\int_{0}^{t_{2}} t_{2}^{1-\gamma} K_{\mu}\left(t_{2}-s\right) f\left(s, u(s), \int_{0}^{s} \rho(s, \tau) h(s, \tau, u(\tau)) d \tau\right) d s \\
& \quad-\int_{0}^{t_{1}} t_{1}^{1-\gamma} K_{\mu}\left(t_{1}-s\right) f\left(s, u(s), \int_{0}^{s} \rho(s, \tau) h(s, \tau, u(\tau)) d \tau\right) d s \\
& \leq \quad\left.\left\|t_{2}^{1-\gamma} S_{v, \mu}\left(t_{2}\right)-t_{2}^{1-\gamma} S_{v, \mu}\left(t_{1}\right)\right\|+\left\|t_{2}^{1-\gamma} S_{v, \mu}\left(t_{1}\right)-t_{1}^{1-\gamma} S_{v, \mu}\left(t_{1}\right)\right\|\right) \\
& \times\left\|\sum_{i=1}^{m} \lambda_{i} I_{0+}^{\gamma} \int_{0}^{\tau_{i}} K_{\mu}\left(\tau_{i}-s\right) f\left(s, u(s), \int_{0}^{s} \rho(s, \tau) h(s, \tau, u(\tau)) d \tau\right) d s\right\| \\
&+\left\|\int_{t_{1}}^{t_{2}} t_{2}^{1-\gamma} K_{\mu}\left(t_{2}-s\right) f\left(s, u(s), \int_{0}^{s} \rho(s, \tau) h(s, \tau, u(\tau)) d \tau\right) d s\right\| \\
&+\| \int_{0}^{t_{1}} t_{1}^{1-\gamma} K_{\mu}\left(t_{2}-s\right) f\left(s, u(s), \int_{0}^{t_{1}} \rho(s, \tau) h(s, \tau, u(\tau)) d \tau\right) d s \\
&+\| \int_{0}^{t_{1}} t_{2}^{1-\gamma} K_{\mu}\left(t_{2}-s\right) f\left(s, u(s), \int_{0}^{s} \rho(s, \tau) h(s, \tau, u(\tau)) d \tau\right) d s \\
&-\int_{0}^{t_{1}} t_{1}^{1-\gamma} K_{\mu}\left(t_{2}-s\right) f\left(s, u(s), \int_{0}^{s} \rho(s, \tau) h(s, \tau, u(\tau)) d \tau\right) d s \| \\
&+I_{2}+I_{3}+I_{4}+I_{5},
\end{aligned}
$$

where

$$
\begin{aligned}
I_{1}= & \left(\left\|t_{2}^{1-\gamma} S_{v, \mu}\left(t_{2}\right)-t_{2}^{1-\gamma} S_{v, \mu}\left(t_{1}\right)\right\|\right) \\
& \times\left\|d \sum_{i=1}^{m} \lambda_{i} I_{0+}^{\gamma} \int_{0}^{\tau_{i}} K_{\mu}\left(\tau_{i}-s\right) f\left(s, u(s), \int_{0}^{s} \rho(s, \tau) h(s, \tau, u(\tau)) d \tau\right) d s\right\|, \\
I_{2}= & \left(\left\|t_{2}^{1-\gamma} S_{v, \mu}\left(t_{1}\right)-t_{1}^{1-\gamma} S_{v, \mu}\left(t_{1}\right)\right\|\right) \\
& \times\left\|d \sum_{i=1}^{m} \lambda_{i} I_{0+}^{\gamma} \int_{0}^{\tau_{i}} K_{\mu}\left(\tau_{i}-s\right) f\left(s, u(s), \int_{0}^{s} \rho(s, \tau) h(s, \tau, u(\tau)) d \tau\right) d s\right\|, \\
I_{3}= & \left\|\int_{t_{1}}^{t_{2}} t_{2}^{1-\gamma} K_{\mu}\left(t_{2}-s\right) f\left(s, u(s), \int_{0}^{s} \rho(s, \tau) h(s, \tau, u(\tau)) d \tau\right) d s\right\|,
\end{aligned}
$$




$$
\begin{aligned}
I_{4}= & \| \int_{0}^{t_{1}} t_{2}^{1-\gamma} K_{\mu}\left(t_{2}-s\right) f\left(s, u(s), \int_{0}^{s} \rho(s, \tau) h(s, \tau, u(\tau)) d \tau\right) d s \\
& -\int_{0}^{t_{1}} t_{1}^{1-\gamma} K_{\mu}\left(t_{2}-s\right) f\left(s, u(s), \int_{0}^{s} \rho(s, \tau) h(s, \tau, u(\tau)) d \tau\right) d s \|, \\
I_{5}= & \| \int_{0}^{t_{1}} t_{1}^{1-\gamma} K_{\mu}\left(t_{2}-s\right) f\left(s, u(s), \int_{0}^{s} \rho(s, \tau) h(s, \tau, u(\tau)) d \tau\right) d s \\
& -\int_{0}^{t_{1}} t_{1}^{1-\gamma} K_{\mu}\left(t_{1}-s\right) f\left(s, u(s), \int_{0}^{s} \rho(s, \tau) h(s, \tau, u(\tau)) d \tau\right) d s \| .
\end{aligned}
$$

Here we calculate

$$
\left\|t_{2}^{1-\gamma}(Q u)\left(t_{2}\right)-t_{1}^{1-\gamma}(Q u)\left(t_{1}\right)\right\| \leq \sum_{i=1}^{5}\left\|I_{i}\right\| .
$$

Therefore, we observe that $\left\|I_{i}\right\|$ tend to 0 , when $t_{2} \rightarrow t_{1}, i=1,2, \ldots, 5$.

For $I_{1}$, by Lemma 2.12, we get

$$
\begin{aligned}
I_{1}= & \left(\left\|t_{2}^{1-\gamma} S_{v, \mu}\left(t_{2}\right)-t_{2}^{1-\gamma} S_{v, \mu}\left(t_{1}\right)\right\|\right) \\
& \times\left\|d \sum_{i=1}^{m} \lambda_{i} I_{0+}^{\gamma} \int_{0}^{\tau_{i}} K_{\mu}\left(\tau_{i}-s\right) f\left(s, u(s), \int_{0}^{s} \rho(s, \tau) h(s, \tau, u(\tau)) d \tau\right) d s\right\| \\
\leq & \left\|t_{2}^{1-\gamma}\left(S_{v, \mu}\left(t_{2}\right)-S_{v, \mu}\left(t_{1}\right)\right)\right\| \\
& \times\left\|d \sum_{i=1}^{m} \lambda_{i} I_{0+}^{\gamma} \int_{0}^{\tau_{i}} K_{\mu}\left(\tau_{i}-s\right) f\left(s, u(s), \int_{0}^{s} \rho(s, \tau) h(s, \tau, u(\tau)) d \tau\right) d s\right\|
\end{aligned}
$$

$\rightarrow 0, \quad$ as $t_{2} \rightarrow t_{1}$.

For $I_{2}$, by Lemma 2.12, we get

$$
\begin{aligned}
I_{2}= & \left(\left\|t_{2}^{1-\gamma} S_{v, \mu}\left(t_{1}\right)-t_{1}^{1-\gamma} S_{v, \mu}\left(t_{1}\right)\right\|\right) \\
& \times\left\|d \sum_{i=1}^{m} \lambda_{i} I_{0+}^{\gamma} \int_{0}^{\tau_{i}} K_{\mu}\left(\tau_{i}-s\right) f\left(s, u(s), \int_{0}^{s} \rho(s, \tau) h(s, \tau, u(\tau)) d \tau\right) d s\right\| \\
\leq & \frac{M b^{1-\gamma}}{\Gamma(v(1-\mu)+\mu)}\left\|t_{2}^{1-\gamma}-t_{1}^{1-\gamma}\right\| \\
& \times\left\|d \sum_{i=1}^{m} \lambda_{i} I_{0+}^{\gamma} \int_{0}^{\tau_{i}} K_{\mu}\left(\tau_{i}-s\right) f\left(s, u(s), \int_{0}^{s} \rho(s, \tau) h(s, \tau, u(\tau)) d \tau\right) d s\right\| \\
\rightarrow & 0, \quad \text { as } t_{2} \rightarrow t_{1} .
\end{aligned}
$$

For $I_{3}$, by Lemma 2.12 and $(\mathrm{H} 2)$, we have

$$
\begin{aligned}
I_{3} & =\left\|\int_{t_{1}}^{t_{2}} t_{2}^{1-\gamma} K_{\mu}\left(t_{2}-s\right) f\left(s, u(s), \int_{0}^{s} \rho(s, \tau) h(s, \tau, u(\tau)) d \tau\right) d s\right\| \\
& \leq \frac{M t_{2}^{1-\gamma}}{\Gamma(\mu)} \int_{0}^{t_{2}}\left(t_{2}-s\right)^{\mu-1} f\left(s, u(s), \int_{0}^{s} \rho(s, \tau) h(s, \tau, u(\tau)) d \tau\right) d s d s
\end{aligned}
$$




$$
=M t_{2}^{1-\gamma} I_{0+}^{\mu} f\left(s, u(s), \int_{0}^{s} \rho(s, \tau) h(s, \tau, u(\tau)) d \tau\right) d s \rightarrow 0, \quad \text { as } t_{2} \rightarrow t_{1} .
$$

For $I_{4}$, by Lemma 2.12 and (H2), we have

$$
\begin{aligned}
I_{4}= & \| \int_{0}^{t_{1}} t_{2}^{1-\gamma} K_{\mu}\left(t_{2}-s\right) f\left(s, u(s), \int_{0}^{s} \rho(s, \tau) h(s, \tau, u(\tau)) d \tau\right) d s \\
& -\int_{0}^{t_{1}} t_{1}^{1-\gamma} K_{\mu}\left(t_{2}-s\right) f\left(s, u(s), \int_{0}^{s} \rho(s, \tau) h(s, \tau, u(\tau)) d \tau\right) d s \| \\
\leq & \frac{2 M}{\Gamma(\mu)} \int_{0}^{t_{1}}\left[t_{2}^{1-\gamma}\left(t_{2}-s\right)^{\mu-1}-t_{1}^{1-\gamma}\left(t_{1}-s\right)^{\mu-1}\right] \\
& \times f\left(s, u(s), \int_{0}^{s} \rho(s, \tau) h(s, \tau, u(\tau)) d \tau\right) d s,
\end{aligned}
$$

then, by the Lebesgue dominated convergence theorem, we have

$$
\begin{aligned}
& \int_{0}^{t_{1}}\left[t_{2}^{1-\gamma}\left(t_{2}-s\right)^{\mu-1}-t_{1}^{1-\gamma}\left(t_{1}-s\right)^{\mu-1}\right] f\left(s, u(s), \int_{0}^{s} \rho(s, \tau) h(s, \tau, u(\tau)) d \tau\right) d s \rightarrow 0, \\
& \quad \text { as } t_{2} \rightarrow t_{1}
\end{aligned}
$$

For $I_{5}$, by Lemma 2.12 and $(\mathrm{H} 2)$, we have

$$
\begin{aligned}
I_{5}= & \| \int_{0}^{t_{1}} t_{1}^{1-\gamma} K_{\mu}\left(t_{2}-s\right) f\left(s, u(s), \int_{0}^{s} \rho(s, \tau) h(s, \tau, u(\tau)) d \tau\right) d s \\
& -\int_{0}^{t_{1}} t_{1}^{1-\gamma} K_{\mu}\left(t_{1}-s\right) f\left(s, u(s), \int_{0}^{s} \rho(s, \tau) h(s, \tau, u(\tau)) d \tau\right) d s \| \\
\leq & \left\|\int_{0}^{t_{1}} t_{1}^{1-\gamma}\left[K_{\mu}\left(t_{2}-s\right)-K_{\mu}\left(t_{1}-s\right)\right] f\left(s, u(s), \int_{0}^{s} \rho(s, \tau) h(s, \tau, u(\tau)) d \tau\right) d s\right\| \\
\leq & \left\|K_{\mu}\left(t_{2}-s\right)-K_{\mu}\left(t_{1}-s\right)\right\| \int_{0}^{t_{1}} t_{1}^{1-\gamma} f\left(s, u(s), \int_{0}^{s} \rho(s, \tau) h(s, \tau, u(\tau)) d \tau\right) d s \\
\rightarrow & 0, \quad \text { as } t_{2} \rightarrow t_{1} .
\end{aligned}
$$

In conclusion,

$$
\left\|t_{2}^{1-\gamma}(Q u)\left(t_{2}\right)-t_{1}^{1-\gamma}(Q u)\left(t_{1}\right)\right\| \rightarrow 0
$$

as $t_{2} \rightarrow t_{1}$, which means that the operator $Q: B_{r} \rightarrow B_{r}$ is equicontinuous.

Let $H=\overline{c o} Q\left(B_{r}\right)$. Then it is easy to verify that $Q$ maps $H$ into itself and $H \subset B_{r}$ is equicontinuous.

Step 4. Now, we prove that $Q: H \rightarrow H$ is a condensing operator. For any $D \subset H$, by Lemma 2.2, there exists a countable set $D_{0}=\left\{u_{n}\right\} \subset D$, such that

$$
\alpha(Q(D)) \leq 2 \alpha\left(Q\left(D_{0}\right)\right)
$$

By the equicontinuity of $H$, we know that $D_{0} \subset D$ is also equicontinuous. 
For $t \in J$, by the definition of $Q$ and (H2)(ii), (H3)(ii), we have

$$
\begin{aligned}
\alpha\left(Q\left(D_{0}\right)(t)\right) & \\
=\alpha & \left(\left\{S_{v, \mu}(t) d \sum_{i=1}^{m} \lambda_{i} I_{0+}^{\gamma} \int_{0}^{\tau_{i}} K_{\mu}\left(\tau_{i}-s\right) f\left(s, u(s), \int_{0}^{s} \rho(s, \tau) h(s, \tau, u(\tau)) d \tau\right) d s\right.\right. \\
& \left.\left.+\int_{0}^{t} K_{\mu}(t-s) f\left(s, u(s), \int_{0}^{s} \rho(s, \tau) h(s, \tau, u(\tau)) d \tau\right) d s\right\}\right) \\
\leq & \frac{2 M}{\Gamma(\mu)} \int_{0}^{t}(t-s)^{\mu-1} \alpha\left(\left\{f\left(s, u(s), \int_{0}^{s} \rho(s, \tau) h(s, \tau, u(\tau)) d \tau\right)\right\}\right) d s \\
\leq & \frac{2 M}{\Gamma(\mu)} \int_{0}^{t}(t-s)^{\mu-1}\left[\eta(s) \alpha\left(D_{0}(u(s))\right)+\varrho \zeta^{*} \xi(t) \alpha\left(D_{0}(u(s))\right)\right] d s \\
\leq & \frac{2 M}{\Gamma(\mu)} \alpha\left(D_{0}(s)\right) \int_{0}^{t}(t-s)^{\mu-1}\left(\eta(s)+\varrho \zeta^{*} \xi(s)\right) d s \\
\leq & \frac{2 M b^{\mu-q}}{\Gamma(\mu)}\left(\frac{1-q}{\mu-q}\right)^{1-q}\left(L_{1}\|\eta\|_{L_{\frac{1}{q}}[0, b]}+L_{2} \varrho \zeta^{*}\|\xi\|_{L_{\frac{1}{q}}[0, b]}\right) \cdot \alpha(D) .
\end{aligned}
$$

Since $Q\left(D_{0}\right) \subset H$ is bounded and equicontinuous, we know from Lemma 2.3 that

$$
\alpha\left(Q\left(D_{0}\right)\right)=\max _{t \in I} \alpha\left(Q\left(D_{0}\right)(t)\right) .
$$

Therefore, we have

$$
\alpha(Q(D)) \leq \frac{4 M b^{\mu-q}}{\Gamma(\mu)}\left(\frac{1-q}{\mu-q}\right)^{1-q}\left(L_{1}\|\eta\|_{L_{\frac{1}{q}}[0, b]}+L_{2} \varrho \zeta^{*}\|\xi\|_{L_{\frac{1}{q}}[0, b]}\right) \leq \alpha(D) .
$$

Thus, $Q: B_{r} \rightarrow B_{r}$ is a condensing operator. It follows from Lemma 2.5 that the problem (1.1) has at least one solution $u \in C_{1-\gamma}(J, E)$. Finally, using [4, Lemma 21] and repeating the process of proof in Lemma 2.8 , one can show that this solution is actually in $C_{1-\gamma}^{\gamma}(J, E)$. This completes the proof.

In the following we will present some special cases.

Case 1. When $B=I$, then $D(B)=E$, we assume that we generate a norm continuous semigroup $\{W(t)\}_{t \geq 0}$ of uniformly bounded linear operators on $E$, then from the proof of Theorem 3.1 we have the following theorem.

Theorem 3.2 Assume that the nonlinear function $f: J \times E^{m} \rightarrow E$ is continuous and the assumptions conditions are satisfies, then for the problem

$$
\left\{\begin{array}{l}
D_{0+}^{v, \mu} u(t)=A u(t)+f\left(t, u(t), \int_{0}^{t} \rho(t, s) h(t, s, u(s)) d s\right), \quad t \in J \\
I_{0+}^{1-\gamma} u(0)=\sum_{i=1}^{m} \lambda_{i} I_{0+}^{(1-v)(1-\mu)} u\left(\tau_{i}\right), \quad \mu \leq \gamma=\mu+v-\mu v
\end{array}\right.
$$

there exists at least one mild solution in $C_{1-\gamma}^{\gamma}(J, E)$.

Case 2. When $B=I, v=1$, then $D(B)=E$, we assume that we generate a norm continuous semigroup $\{W(t)\}_{t \geq 0}$ of uniformly bounded linear operator on $E$, then from the proof of Theorem 3.1 we have the following theorem. 
Theorem 3.3 Assume that the nonlinear function $f: J \times E^{m} \rightarrow E$ is continuous and the assumptions conditions are satisfied, then for the problem

$$
\left\{\begin{array}{l}
{ }^{C} D_{0+}^{\mu} u(t)=A u(t)+B f\left(t, u(t), \int_{0}^{t} \rho(t, s) h(t, s, u(s)) d s\right), \quad t \in J, \\
u(0)=\sum_{i=1}^{m} \lambda_{i} u\left(\tau_{i}\right)
\end{array}\right.
$$

there exists at least one mild solution in $C(J, E)$.

Case 3. When $v=1$, then $D(B)=E$, we assume that we generate a norm continuous semigroup $\{W(t)\}_{t \geq 0}$ of uniformly bounded linear operator on $E$, then from the proof of Theorem 3.1 we have the following theorem.

$$
\left\{\begin{array}{l}
{ }^{C} D_{0+}^{\mu} B u(t)=A u(t)+B f\left(t, u(t), \int_{0}^{t} \rho(t, s) h(t, s, u(s)) d s\right), \quad t \in J, \\
B u(0)=\sum_{i=1}^{m} \lambda_{i} B u\left(\tau_{i}\right) .
\end{array}\right.
$$

Proof Let $u_{0}-g(u)=\sum_{i=1}^{m} \lambda_{i} u\left(\tau_{i}\right)$, then Eq. (3.5) is transformed to the following equation:

$$
\left\{\begin{array}{l}
{ }^{C} D_{0+}^{\mu} B u(t)=A u(t)+B f\left(t, u(t), \int_{0}^{t} \rho(t, s) h(t, s, u(s)) d s\right), \quad t \in J \\
B u(0)=B\left(u_{0}-g(u)\right) .
\end{array}\right.
$$

For the problem (3.6), for more details see [6].

\section{Applications}

In this section, we give one example to illustrate our main results derived in Sect. 3.

\section{Example 4.1}

We consider the following fractional diffusion equations of Sobolev type with delay:

$$
\left\{\begin{array}{l}
D_{0+}^{v, \mu}\left(u(t, x)-\frac{\partial^{2} u(t, x)}{\partial x^{2}}\right)=\frac{\partial^{2}}{\partial x^{2}} u(t, x)+\widetilde{f}\left(t, u(t, x), \int_{0}^{t} \rho(t, s) h(t, s, u(s, x)) d s\right) \\
\quad x \in \Omega, t \in(0,1] \\
I_{0+}^{1-\gamma}\left(u(0, x)-\frac{\partial^{2}}{\partial x^{2}} u(0, x)\right)=\widetilde{\varphi}(x) \\
\quad x \in \Omega
\end{array}\right.
$$

where $D_{0+}^{v, \mu}$ is the Hilfer fractional derivative, $0 \leq v \leq 1,0<\mu<1, \tau_{k}: J \rightarrow J$ are continuous functions such that $0 \leq \tau_{k}(t)<t, k=1,2, \ldots, m$ and $\Omega \subset R^{m}$ is a bounded domain with a sufficiently smooth boundary $\partial \Omega$, and $\tilde{f}: J \times R^{m} \rightarrow R$ is continuous.

Let $E=L^{2}(\Omega)$ be a Banach space with the $L^{2}$-norm $\|\cdot\|_{2}$, we define

$$
D(A)=D(B)=H^{2}(\Omega), \quad A u=\frac{\partial^{2} u}{\partial x^{2}}, \quad B u=u-\frac{\partial^{2} u}{\partial x^{2}},
$$

where $H^{2}(\Omega)$ is the completion of the space $C^{2}(\Omega)$ with respect to the norm

$$
\|u\|_{H^{2}(\Omega)}=\left(\int_{\Omega} \sum_{|\mu| \leq 2}\left|D^{\mu} u(x)\right|^{2} d x\right)^{\frac{1}{2}}
$$


Fou and Li Boundary Value Problems (2018) 2018:48

Page 22 of 25

$C^{2}(\Omega)$ is the set of all continuous defined on $R$ which have continuous partial derivatives of order less than or equal to 2 . In view of the paper [19], it is easy to see that the pair $(A, B)$ generates a propagation family $W(t)$ of uniformly bounded, and similarly to the proof of (2.15), (2.16) and (2.17) in [19], we can see that $\{W(t)\}_{t \geq 0}$ is norm continuous for $t>0$ and $\|W(t)\| \leq 1$, i.e., the assumption condition (H1) is satisfied.

Let $v=\frac{1}{2}, \mu=\frac{1}{2}$, then $\gamma=\frac{3}{4}$ and

$$
\begin{aligned}
& \widetilde{f}\left(t, u(t, x), \int_{0}^{t} \rho(t, s) h(t, s, u(s, x)) d s\right)=B f\left(t, u(t, x), \int_{0}^{t} \rho(t, s) h(t, s, u(s, x)) d s\right) \\
& =\frac{1}{k \cdot \sqrt[k]{t}} u(t, x)+\frac{t^{2}}{k} \int_{0}^{t} s^{2} \cdot \sin \frac{u(s, x)}{t} d s, \\
& \widetilde{\varphi}(\cdot)=2 I_{0+}^{\gamma} B\left(u\left(\frac{1}{2}, x\right)-\frac{\partial^{2}}{\partial x^{2}} u\left(\frac{1}{2}, x\right)\right), \\
& \rho(t, s)=1 \text {, } \\
& h(t, s, u(s, x))=s^{2} \cdot \sin \frac{u(s, x)}{t} .
\end{aligned}
$$

Equation (4.1) can be rewritten in the abstract form as (1.1). Moreover,

$$
\begin{aligned}
& \left\|\widetilde{f}\left(t, u(t, x), \int_{0}^{t} \rho(t, s) h(t, s, u(s, x)) d s\right)\right\| \\
& \quad \leq \frac{1}{k \cdot \sqrt[k]{t}}\|u(t, x)\|+\frac{t^{2}}{k}\left\|\int_{0}^{t} h(t, s, u(s, x)) d s\right\| \\
& \quad:=\mu_{1}(t)\|u(t, x)\|+\mu_{2}(t)\left\|\int_{0}^{t} h(t, s, u(s, x)) d s\right\|,
\end{aligned}
$$

and, for any $u_{1}, u_{2} \in E$,

$$
\begin{aligned}
& \left\|\tilde{f}\left(t, u_{1}(t, x), \int_{0}^{t} \rho(t, s) h\left(t, s, u_{1}(s, x)\right) d s\right)-\tilde{f}\left(t, u_{2}(t, x), \int_{0}^{t} \rho(t, s) h\left(t, s, u_{2}(s, x)\right) d s\right)\right\| \\
& \quad \leq \frac{1}{k \cdot \sqrt[k]{t}}\left\|u_{1}(t, x)-u_{2}(t, x)\right\|+\frac{t^{2}}{k}\left\|\int_{0}^{t} h\left(t, s, u_{2}(s, x)\right) d s-\int_{0}^{t} h\left(t, s, u_{1}(s, x)\right) d s\right\| .
\end{aligned}
$$

Therefore, for any bounded set $D_{1}, D_{2} \in E$,

$$
\left.\alpha \tilde{f}\left(t, D_{1}, D_{2}\right)\right) \leq \frac{1}{k \cdot \sqrt[k]{t}}\left(\alpha\left(D_{1}\right)\right)+\frac{t^{2}}{k}\left(\alpha\left(D_{2}\right)\right):=\eta(t)\left(\alpha\left(D_{1}\right)\right)+\xi(t)\left(\alpha\left(D_{1}\right)\right), \quad t \in(0,1] .
$$

Moreover,

$$
\|h(t, s, u(s, x))\| \leq \frac{s^{2}}{t}\|u(s, x)\|:=m(t, s)\|u(s, x)\|
$$

and

$$
\sup _{t \in[0,1]} \int_{0}^{t} m(t, s) d s=\sup _{t \in[0,1]} \int_{0}^{t} \frac{s^{2}}{t} d s=\frac{1}{3}:=m^{*}
$$


For any $u_{1}, u_{2} \in E$,

$$
\begin{aligned}
\left\|h\left(t, s, u_{1}(s, x)\right)-h\left(t, s, u_{2}(s, x)\right)\right\| & \leq \frac{s^{2}}{t}\|u(s, x)\|:=m(t, s)\|u(s, x)\| \\
& \leq \frac{s^{2}}{t}\left\|u_{1}(s, x)-u_{2}(s, x)\right\| .
\end{aligned}
$$

So, for any bounded set $D_{1} \subset E$,

$$
\alpha\left(h\left(t, s, D_{1}\right)\right) \leq \frac{s^{2}}{t} \alpha\left(D_{1}\right): \zeta(t, s) \alpha\left(D_{1}\right)
$$

and

$$
\sup _{t \in[0,1]} \int_{0}^{t} \zeta(t, s) d s=\sup _{t \in[0,1]} \int_{0}^{t} \frac{s^{2}}{t} d s=\frac{1}{3}:=\zeta^{*}
$$

If we put $q=\frac{1}{4}, k=5, M=1, L_{1}=L_{2}=\frac{1}{2}, \tau_{i}=\frac{1}{2}, b=1, \sum_{i=1}^{m}\left|\lambda_{i}\right|=2$, then

$$
\begin{aligned}
& \left\|\mu_{1}\right\|_{L_{\frac{1}{q}}[0, b]}=\|\eta\|_{L_{\frac{1}{q}}[0, b]}=\left(\frac{1}{5}\right)^{\frac{3}{4}}, \quad \mu_{2}^{*}=\frac{1}{5} \\
& \left\|\mu_{2}\right\|_{L_{\frac{1}{q}}[0, b]}=\|\xi\|_{L_{\frac{1}{q}}[0, b]}=\left(\frac{1}{5}\right)^{\frac{3}{2}} .
\end{aligned}
$$

On the other hand,

$$
|d|=\left|\frac{1}{1-\sum_{i=1}^{m} \lambda_{i} I_{0+}^{\gamma} S_{v, \mu}\left(\tau_{i}\right)}\right|=\left|\frac{1}{1-\frac{2 \sqrt{2}}{\sqrt{\pi}}}\right| \approx 1.6785
$$

and we have

$$
\begin{gathered}
\frac{M^{2}|d|}{\Gamma(\gamma) \Gamma(\mu+\gamma)} \sum_{i=1}^{m}\left|\lambda_{i}\right|\left[\left(\frac{1-q}{\mu+\gamma-q}\right)^{1-q} \tau_{i}^{\mu+\gamma-q}\left\|\mu_{1}\right\|_{L_{\frac{1}{q}}[0, b]}+\frac{b^{\mu+\gamma} \varrho m^{*} \mu_{2}^{*}}{\mu+\gamma}\right] \\
+\frac{M b^{1-\gamma}}{\Gamma(\mu)}\left[\left(\frac{1-q}{\mu-q}\right)^{1-q} b^{\mu-q}\left\|\mu_{1}\right\|_{L_{\frac{1}{q}}[0, b]}+\frac{b^{\mu} \varrho m^{*} \mu_{2}^{*}}{\mu}\right] \approx 0.985282<1
\end{gathered}
$$

and

$$
\frac{M b^{\mu-q}}{\Gamma(\mu)}\left(\frac{1-q}{\mu-q}\right)^{1-q}\left(L_{1}\|\eta\|_{L_{\frac{1}{q}}[0, b]}+L_{2} \varrho \zeta^{*}\|\xi\|_{L_{\frac{1}{q}}[0, b]}\right) \approx 0.211485<\frac{1}{4}
$$

Now all the assumptions in Theorem 3.1 are satisfied, Eq. (4.1) has at least one solution in $C_{\frac{1}{4}}(J)$.

\section{Conclusions}

In this paper, we deal with a class of nonlinear fractional differential equations of Sobolev type with boundary conditions by using the Hilfer fractional derivative, which generalizes the famous Riemann-Liouville fractional derivative. With the help of the properties of Hilfer fractional calculus, the theory of propagation families as well as the theory of the 
measure of noncompactness and the fixed point methods, we obtain the existence result of mild solutions for Sobolev-type fractional evolution differential equations. Finally, an example is presented to illustrate the main result.

\section{Acknowledgements}

The authors are grateful to anonymous referees for their constructive comments and suggestions, which have greatly improved this paper.

Funding

This work is supported by National Natural Science Foundation of China (Grant No. 11061031).

\section{Competing interests}

The authors declare that they have no competing interests.

\section{Consent for publication}

Both authors read and approved the final version of the manuscript.

\section{Authors' contributions}

All authors contributed equally to the writing of this paper. All authors read and approved the final manuscript.

\section{Publisher's Note}

Springer Nature remains neutral with regard to jurisdictional claims in published maps and institutional affiliations.

Received: 28 November 2017 Accepted: 22 March 2018 Published online: 03 April 2018

\section{References}

1. Agarwal, R.P., Benchohra, M., Hamani, S.: A survey on existence result for boundary value problem of nonlinear fractional differential equations and inclusions. Acta Appl. Math. 109, 973-1033 (2010)

2. Aghajani, A., Banaś, J., Sabzali, N.: Some generalizations of Darbo fixed point theorem and application. Bull. Belg. Math. Soc. Simon Stevin 20(2), 345-358 (2013)

3. El-Borai, M.M.: The fundamental solutions for fractional evolution equations of parabolic type. J. Appl. Math. Stoch. Anal. 3, 197-211 (2004)

4. Furati, K.M., Kassim, M.D., Tatar, N.E.: Existence and uniqueness for a problem involving Hilfer factional derivative. Comput. Math. Appl. 64, 1612-1626 (2012)

5. Gu, H., Trujillo, J.J.: Existence of mild solution for evolution equation with Hilfre fractional derivative. Appl. Math. Comput. 257, 344-354 (2015)

6. Li, F., Liang, J., Xu, H.K.: Existence of mild solutions for fractional integrodifferential equations of Sobolev type with nonlocal conditions. J. Math. Anal. Appl. 391, 510-525 (2012)

7. Lakzian, H., Gopal, D., Sintunavarat, W.: New fixed point results for mappings of contractive type with an application to nonlinear fractional differential equations. J. Fixed Point Theory Appl. https://doi.org/10.1007/s11874-015-0275-7

8. Mainardi, F., Ponce, R., Gorenflo, R.: Probability distributions generated by fractional diffusion equations. In: Kondor, K.I. (ed.) Econorhysics: App. Math. Comput., vol. 216, pp. 61-69 (2010)

9. Hilfer, R.: Applications of Fractional Calculus in Physics. World Scientific, Singapore (2000)

10. Bachar, I., Maagli, H., Radulescu, V.: Positive solutions for superlinear Riemann-Liouville fractional boundary-value problems. Electron. J. Differ. Equ. 2017, 240 (2017)

11. Denton, Z., Ramírez, J.D.: Existence of minimal and maximal solutions to RL fractional integro-differential initial value problems. Opusc. Math. 37(5), 705-724 (2017)

12. Guner, O., Bekir, A., Bilgil, H.: A note on exp-function method combined with complex transform method applied to fractional differential equations. Adv. Nonlinear Anal. 4(3), 201-208 (2015)

13. Idczak, D., Walczak, S.: On a linear-quadratic problem with Caputo derivative. Opusc. Math. 36(1), 49-68 (2016)

14. Molica Bisci, G., Repovs, D.: Multiple solutions of $p$-biharmonic equations with Navier boundary conditions. Complex Var. Elliptic Equ. 59(2), 271-284 (2014)

15. Torres Ledesma, C.: Boundary value problem with fractional p-Laplacian operator. Adv. Nonlinear Anal. 5(2), $133-146$ (2016)

16. Xiang, M., Zhang, B., Radulescu, V.: Existence of solutions for perturbed fractional $p$-Laplacian equations. J. Differ. Equ. 260(2), 1392-1413 (2016)

17. Zhang, X., Zhang, B., Repovs, D.: Existence and symmetry of solutions for critical fractional Schrödinger equations with bounded potentials. Nonlinear Anal. 142,48-68 (2016)

18. Balachandran, K., Kiruthika, S.: Existence of solutions of abstract fractional impulsive semilinear evolution equations. Electron. J. Qual. Theory Differ. Equ. 2010, 4 (2010)

19. Liang, J., Xiao, T.J.: Abstract degenerate Cauchy problems in locally convex spaces. J. Math. Anal. Appl. 259, 398-412 (2001)

20. Wang, G., Zhang, L., Song, G.: Systems of first order impulsive functional differential equations with deviating arguments and nonlinear boundary conditions. Nonlinear Anal. 74, 974-982 (2011)

21. Ahmad, B., Sivasundaram, S.: Existence results for nonlinear impulsive hybrid boundary value problems involving fractional differential equations. Nonlinear Anal. Hybrid Syst. 3, 251-258 (2009)

22. Benchohra, M., Seba, D.: Impulsive fractional differential equations in Banach spaces. Electron. J. Qual. Theory Differ. Equ. 2009, 8 (2009) 
23. Benchohra, M., Henderson, J., Ntouyas, S.: Impulsive Differential Equations and Inclusions. Contemporary Mathematics and Its Applications, vol. 2. Hindawi, Cairo (2006)

24. Li, K., Peng, J., Jia, J.: Cauchy problems for fractional differential equations with Riemann-Liouville fractional derivatives. J. Funct. Anal. 263, 476-510 (2012)

25. Liu, Y.: Existence of solutions for impulsive differential models on half lines involving Caputo fractional derivatives. Commun. Nonlinear Sci. Numer. Simul. 18, 2604-2625 (2013)

26. Zhou, Y., Jiao, F.: Nonlocal Cauchy problem for fractional evolution equations. Nonlinear Anal., Real World Appl. 11, 4465-4475 (2010)

27. Hernández, E., O'Regan, D., Balachandran, K.: On recent developments in the theory of abstract differential equations with fractional derivatives. Nonlinear Anal. 73, 3462-3471 (2010)

28. Cabada, A., Hamdi, Z: Nonlinear fractional differential equations with integral boundary value conditions. Appl. Math. Comput. 228, 251-257 (2014)

29. Wang, J., Fečkan, M., Zhou, Y.: On the new concept of solutions and existence results for impulsive fractional evolution equations. Dyn. Partial Differ. Equ. 8, 345-361 (2011)

30. Kumar, S., Sukavanam, N.: Approximate controllability of fractional order semilinear systems with bounded delay. J. Differ. Equ. 252, 6163-6174 (2012)

31. Stamova, l.: Global stability of impulsive fractional differential equations. Appl. Math. Comput. 237, 605-612 (2014)

32. Hilfer, R., Luchko, Y., Tomovski, Ž.: Operational method for the solution of fractional differential equations with generalized Riemann-Liouville fractional derivatives. Fract. Calc. Appl. Anal. 12(3), 299-318 (2009)

33. Hilfer, R.: In: Hilfer, R. (ed.) Fractional Time Evolution, Applications of Fractional Calculus in Physics, pp. 87-130. World Scientific, Singapore (2000)

34. Gou, H., Li, B.: Existence of mild solutions for fractional nonautonomous evolution equations of Sobolev type with delay. J. Inequal. Appl. 2017, 252 (2017)

35. Wang, J., Zhang, Y.: Nonlocal initial value problems for differential equations with Hilfer fractional derivative. Appl. Math. Comput. 266, 850-859 (2015)

36. Gao, Z.Y., Yu, X.L.: Existence results for BVP of a class of Hilfer fractional differential equations. J. Appl. Math. Comput. https://doi.org/10.1007/s12190-016-1070-3

37. Lightbourne, J.H., Rankin, S.M.: A partial functional differential equation of Sobolev type. J. Math. Anal. Appl. 93 328-337 (1983) 245, 74-85 (2014)

38. Kerboua, M., Debbouche, A., Baleanu, D.: Approximate controllability of Sobolev type fractional stochastic nonlocal nonlinear differential equations in Hilbert spaces. Electron. J. Qual. Theory Differ. Equ. 58, 1 (2014)

39. Ponce, R.: Holder continuous solutions for Sobolev type differential equations. Math. Nachr. 287, 70-78 (2014)

40. Agarwal, R., Meehan, M., O'Regan, D.: Fixed Point Theory and Applications. Cambridge University Press, Cambridge (2001)

41. Guo, D., Cho, Y., Zhu, J.: Partial Ordering Methods in Nonlinear Problems. NOVA Publ., New York (2004)

42. Li, Y.X.: The positive solutions of abstract semilinear evolution equations and their applications. Acta Math. Sin. 39(5), 666-672 (1996) (in Chinese)

43. Banas, J., Goebel, K.: Measure of Noncompactness in Banach Spaces. Lect. Notes Pure Appl. Math., vol. 60. Dekker, New York (1980)

44. El-Borai, M.M.: Some probability densities and fundamental solutions of fractional evolution equations. Chaos Solitons Fractals 14, 433-440 (2002)

45. Deimling, K.: Nonlinear Functional Analysis. Springer, New York (1985)

46. Kilbas, A.A., Srivastava, H.M., Trujillo, J.J.: Theory and Applications of Fractional Differential Equations. Elsevier, Amsterdam (2006)

\section{Submit your manuscript to a SpringerOpen ${ }^{\circ}$ journal and benefit from:}

- Convenient online submission

- Rigorous peer review

- Open access: articles freely available online

- High visibility within the field

- Retaining the copyright to your article

Submit your next manuscript at $\gg$ springeropen.com 